\title{
Les grands enjeux : les secteurs industriels
}

La mécanique est la pierre angulaire de toutes les industries. Elle est à la base de la création et de la fabrication de tous les objets technologiques ou communs qui nous entourent.

Pour mieux convaincre le lecteur de cette assertion, ce chapitre a pour but de montrer les grands enjeux auxquels sont confrontés des secteurs industriels importants de notre économie.

\subsection{L'industrie automobile}

\subsubsection{Importance macroéconomique du secteur}

La mobilité est devenue un droit et, dans ce cadre, l'automobile reste une composante majeure de la vie quotidienne. Objet de rêve tant qu'elle n'est restée accessible qu'à une minorité, elle a perdu progressivement son caractère magique. Sa généralisation, par les contraintes qu'elle entraîne, a mis en lumière des impacts pour la société : pollution, congestion, accidentologie.

En France, l'ind ustrie automobile avec ses constructeurs (deux nationaux Renault et PSA, plus Toyota) et ses équipementiers (Valeo, Faurecia, Plastic Omnium...) reste et restera une industrie majeure de notre pays, malgré les difficultés auxquelles elle est confrontée. Elle représente 2,4 millions d'emplois (25\% fabrication, $25 \%$ services, $50 \%$ transports), soit $10 \%$ de l'emploi direct et indirect en France.

L'industrie automobile est en pleine mutation, devant faire face au défi de la croissance par un marché tiré par les pays émergents (Brésil, Russie, Inde, Chine), au défi de la concurrence en termes de modèles (haut de gamme versus low cost), au défi de la compétitivité (fabriquer localement auprès des marchés), aux défis énergétiques et environnementaux (véhicules électriques ou hybrides, pile à combustible, hydrogène, pièges à NOx...).

Cette industrie doit garder une importance stratégique, en s'appuyant sur les réflexions de la Plateforme de la filière automobile mise en place en 2009, pour développer :

- la recherche, l'innovation, les techniques et la normalisation associée ;

- les métiers, les compétences et les formations ; 
- l'excellence industrielle ;

- les stratégies et les mutations.

\subsubsection{Tendances, enjeux et défis du secteur à l'horizon 2020}

Dans le cadre d'une philosophie du retour à l'essentiel liée peut-être à une perte de confiance dans le progrès technique, l'automobile se trouve confrontée au plus grand défi technologique depuis 80 ans. Un foisonnement de solutions techniques pour réduire l'empreinte $\mathrm{CO}_{2}$ des véhicules, intégrant leur fabrication, entraîne l'industrie automobile dans de multiples voies dont la convergence est incertaine. Il est nécessaire de concevoir, de fabriquer et de vendre dans le cadre d'une approche par le cycle de vie, allant des matières premières à la valorisation du produit usagé, en passant par la fabrication, la distribution et l'utilisation du produit.

Au-delà de la mondialisation qui conduira toujours plus à concevoir et fabriquer auprès de ses marchés, les objectifs techniques à l'horizon 2020 concernent :

- le développement de systèmes de motorisation propre, avec l'étude d'un scénario "limité » (continuité des solutions actuelles) et d'un scénario de « rupture " (évolutions radicales), et cela dans le cadre de deux voies : l'amélioration de moteurs à combustion interne et le développement de véhicules hybrides/électriques ;

- des niveaux de consommation accessibles (ou d'autonomie pour un véhicule électrique) par ses caractéristiques masse, dimension, SCx, résistance au roulement;

- la trace écologique du véhicule par les matériaux employés et leur origine, leur aptitude au démontage et leur recyclabilité, leur coût « $\mathrm{CO}_{2}$ » de mise en œuvre et l'efficience énergétique des moyens de production ;

- les synthèses véhicule/chaîne de traction/prestations recherchées qui peuvent permettre des équilibres différents ;

- l'acceptabilité de l'automobile en termes de sécurité, en particulier par son caractère non agressif ;

- une conduite apaisée, agréable et confortable par les progrès apportés en matière de silence, de véhicule communiquant, de conduite assistée ou aidée, gage à la fois de la compétitivité de l'offre automobile et de sa bonne contribution à la sécurité globale.

\subsubsection{Attentes vis-à-vis des partenaires-fournisseurs équipementiers-ensembliers}

Si les constructeurs sont responsables des prestations et de la qualité finale des véhicules qu'ils conçoivent et qu'ils produisent, ils dépendent pour une majorité de composants des équipementiers. La maîtrise et le contrôle de la chaîne des 
fournisseurs est d'autant plus essentielle que la décentralisation de la conception et de la fabrication est une donnée a priori irréversible. Il faut en particulier :

- pouvoir communiquer en temps réel et donc avoir les mêmes outils de modélisation et de simulation tout au long de cette chaîne ;

- pouvoir fabriquer au plus près de l'usine de montage du constructeur, pour éviter les problèmes de transport de pièces ou d'ensembles de composants ;

- être capable, à partir d'un cahier des charges fonctionnel, de proposer des solutions innovantes, d'autant plus attendues que les fournisseurs maîtrisent mieux que le constructeur les composants qu'ils conçoivent et fabriquent.

L'optimisation du ratio coût/valeur, par la réduction des coûts de production, la standardisation, la réduction des investissements, la réduction des délais de développement (time to market des innovations), ne peut s'obtenir qu'avec la participation active et partagée des équipementiers/fournisseurs dans le monde entier.

\subsubsection{Domaines clés de recherche et innovation}

L'électronique a envahi l'automobile, que ce soit dans le domaine de la sécurité active, dans le confort, l'assistance à la conduite, l'interface Homme/machine... Mais l'objet automobile reste un produit mécanique de base, auquel on associe l'électronique pour former des produits «mécatroniques », en particulier tous les capteurs et actuateurs intégrés dans le fonctionnement des véhicules.

Indépendamment de cet aspect, on peut retenir que les grands domaines de la R\&D de la mécanique sont les suivants :

- réduction de la masse : cette réduction concerne l'utilisation de matériaux à faible densité, en particulier les composites et les alliages d'aluminium ou de magnésium pour la caisse en blanc, les polymères pour les vitrages. Les problèmes principaux à résoudre sont la connaissance des lois de comportement de ces nouveaux matériaux, la simulation de leurs procédés de mise en œuvre, la connaissance de leurs propriétés après transformation et surtout le problème de l'assemblage de matériaux différents, y compris la simulation de ces assemblages ;

- amélioration de l'aérodynamique : cette amélioration concerne les traitements locaux (dessous de caisse, rétroviseurs, passages de roues...), avec l'usage de codes de mécanique des fluides de plus en plus performants pour traiter ces particularités ;

- réduction de la consommation des équipements : cette réduction influe directement sur la consommation du véhicule et est en particulier fondamentale pour l'augmentation de l'autonomie des véhicules électriques. Les principaux équipements qui méritent ce type de développement sont le chauffage, la climatisation, le dégivrage/désembuage, l'essuyage, l'assistance de direction ;

- nouvelles motorisations : le court terme concerne l'amélioration des motorisations classiques, en travaillant sur les rendements thermodynamiques, la mécanique des fluides pour la suralimentation, la réduction des frottements 
par l'utilisation de nouveaux revêtements et le traitement acoustique. Le plus long terme consiste à développer des véhicules électriques/hybrides avec des travaux sur les moteurs électriques et le système d'intégration avec gestion thermique et gestion électronique de contrôle et de sécurité ;

- écoconception : ce domaine est étroitement lié à l'utilisation de matériaux soit recyclés, soit biosourcés. Doit être posée la question de l'évaluation de leurs performances qui est souvent coûteuse et limite leurs applications. Il est aussi en relation avec la réutilisation de pièces ou d'ensembles fonctionnels comme pièces de rechange, avec la même question de l'évaluation de leurs performances ;

- écofabrication : il faut limiter les coûts de fabrication et d'assemblage, et limiter les rebuts de production, par une modélisation de la chaîne de fabrication prenant en compte tous les paramètres ;

- analyse du cycle de vie : c'est l'outil de mesure environnemental reconnu par tous. Mais son application est contestée par la non-fiabilité des données d'entrée, par la liberté dans le choix des hypothèses, par la complexité de la méthode difficilement applicable par les PME... Il faut arriver à un logiciel de référence dont la nature des résultats ne soit pas contestée ;

- modélisation et simulation : c'est ce domaine qui a fait les plus grands progrès ces dernières années et qui a changé la structure et le savoir-faire des bureaux d'études et de méthodes. Il n'en reste pas moins que de nombreux progrès sont encore nécessaires pour assurer la continuité de la démarche de l'amont à l'aval, prendre en compte les incertitudes sur les données d'entrée et la dispersion des moyens de production. Il faut des outils qui permettent de concevoir pour produire robuste, conformément au cahier des charges imposé ;

- réalité virtuelle et augmentée : c'est l'environnement actuel du styliste et concepteur, mais peu appliqué à la recherche de solutions mécaniques. Il ne s'agit pas de recherche à proprement parler, mais plus de l'application de solutions existantes à de nouveaux problèmes. Cet aspect peut aussi être relié au prototypage rapide pour valider rapidement des solutions virtuelles.

\subsection{L'industrie aéronautique et spatiale}

\subsubsection{Importance macroéconomique du secteur}

Voler, imiter les oiseaux, un rêve que nous avons tous fait. Ce rêve a poussé d'ingénieux inventeurs à prendre des risques inouïs, parfois au péril de leur vie. Le développement des avions, des hélicoptères, des fusées n'a cessé d'améliorer la performance et la sécurité de ces machines, et de permettre à l'Homme de satisfaire ses rêves, ses envies et ses besoins grandissants.

Depuis 1946, la courbe de croissance du trafic aérien ressemble à la trajectoire d'un avion en montée rapide. De 108 millions en 1960, le nombre de passagers est passé à 1 milliard au début des années 1990, puis à 2,9 milliards en 2012. D'après les analyses des spécialistes du secteur, le cap des 3 milliards sera franchi en 2013. 
D'après l'OACI (Organisation de l'Aviation Civile Internationale), le trafic devrait dépasser les 6 milliards de passagers d'ici 2030, avec un taux de croissance de $5 \%$ par an.

En 2012, le transport aérien représentait environ $35 \%$ en valeur des échanges commerciaux et a généré, à l'échelle mondiale, plus de 5,5 millions d'emplois directs (industrie manufacturière, aéroports et compagnies aériennes) et plus de 26 millions d'emplois indirects (services, tourisme, etc.) [1, 4]. Actuellement, pour l'Europe, cela signifie 500000 emplois directs et un chiffre d'affaires d'environ 130 milliards d'euros soit $30 \%$ du chiffre d'affaires mondial [8]. Les acteurs majeurs de ces domaines sont pour l'Europe: Airbus, ATR, Dassault Aviation, Eurocopter, Agusta Westland, Safran, Rolls Royce, Thales, BAE Systems, Finmeccanica, Saab, Avio... Il est important pour les analyses de considérer l'ensemble du cycle de vie du produit du point de vue du coût de possession, de la réparation et du recyclage, et donc des acteurs spécifiques impliqués. Du fait de leur nombre, ils ne sont pas explicitement cités dans cet article, mais leur liste est disponible.

Le secteur spatial européen a représenté en 2012 environ 6,5 milliards d'euros de chiffre d'affaires (consolidé) et procuré plus de 35000 emplois directs dont 13000 en France $[9,10]$. Ces chiffres ne prennent pas en compte l'activité liée aux missiles stratégiques. Les acteurs majeurs du secteur sont Airbus Defence and Space, Thales Alenia Space, Finmeccanica, OHB, Ruag et Safran.

Les projections sont-elles crédibles ? Il est de notoriété publique que l'aéronautique et le spatial sont des secteurs cycliques de l'industrie (quel domaine ne l'est pas ?). Il n’y a qu'à regarder la courbe de croissance du trafic aérien [3, 4, 7] qui montre régulièrement des stagnations voire des baisses en réaction à des phénomènes économiques ou politiques mondiaux (chocs pétroliers, guerres du Golfe, crises financières...). Il n'en reste pas moins que cette courbe est macroscopiquement en perpétuelle croissance depuis le début.

Pour l'heure, en particulier pour le secteur aéronautique, tous les ingrédients d'une nouvelle période de croissance sont en place. À commencer par le principal : la croissance de l'économie mondiale [2]. D'après les statistiques, 1 point de croissance du PIB mondial représente 1,5 point de hausse du trafic aérien. Compte tenu du fait que les prévisions les plus basses projettent une croissance de l'économie mondiale de $3 \%$ par an, le trafic aérien devrait bien doubler sur les vingt prochaines années. Cette progression devrait être trois fois plus rapide [2] dans les pays émergents. Les prévisions d'Airbus, de Boeing et de l'IATA prévoient également une croissance de 4,6 entre 2012 et 2030 [2, 3, 4, 5, 6, 7] pour l'Inde et la Chine. En effet, ces pays sont en pleine croissance économique et en pleine évolution sociétale qui entraînent des besoins de transports accrus. Or, ces pays ne sont pas encore équipés d'infrastructures terrestres capables de répondre à des besoins croissants. Seul le transport aérien est capable de répondre rapidement à cette demande avec des coûts acceptables pour ces pays.

À ce moteur principal, il faut ajouter le nombre croissant de migrants internationaux (190 millions en 1990 pour 240 projetés en 2013 [2]), la croissance du tourisme et l'augmentation de la classe moyenne mondiale dont l'effectif devrait passer de 2,1 à 5 milliards d'ici 2030 [2]. 
Toutes les analyses montrent que la croissance du trafic aérien est durable. La communauté mécanicienne est indispensable pour le soutien de cette croissance et doit tout faire pour réussir les développements nécessaires en matière d'aéronefs, d'infrastructures et d'organisation pour être capable de répondre à ces besoins dans le respect de l'environnement, tout en assurant la compétitivité des produits et des entreprises du secteur.

Quant au secteur spatial européen, il se répartit entre le domaine institutionnel, dominé par l'Agence spatiale européenne, et le secteur commercial. Ce dernier étant principalement constitué de ventes aux opérateurs privés de satellites et de ventes de lanceurs à Arianespace. Les perspectives de croissance à moyen terme sont faibles dans le contexte économique occidental actuel. L'effet bénéfique de la croissance des pays émergents sera contrebalancé par l'apparition de compétiteurs nouveaux sur les marchés à l'export (vente de satellites et/ou de services de lancement).

\subsubsection{Tendances, enjeux et défis du secteur à horizon 2020}

Les domaines aéronautiques et spatiaux sont caractérisés par des temps de développement et d'exploitation longs (5 à 10 ans pour les développements et 10 à 40 ans pour l'exploitation), par la complexité élevée des produits et la nécessité d'atteindre de très hauts niveaux de performance. Il faut y ajouter un niveau d'exigence très élevé du point de vue de la sécurité et de la sûreté de fonctionnement, qui impose un référentiel de certification très contraignant et un niveau de qualité et de contrôle très exigeant. Il faut enfin considérer qu'en opposition avec d'autres domaines techniques, l'aéronautique est paradoxalement un marché que l'on peut qualifier de niche du fait des tailles des séries. En effet, rares sont les modèles d'avions construits à des cadences supérieures à 15 par mois (à l'exception des avions monocouloir construits à une cadence de 40 par mois). Pour le spatial, les cadences sont encore plus basses puisque les produits les plus récurrents (lanceurs) ne dépassent pas 7 modèles par an. Cela implique des exigences d'anticipation sur la maturité (technique et industrielle) des produits lors de leur introduction en service, des exigences en matière d'outils de conception (design, analyse numérique, etc.) et de justification réglementaire (essais, mesures, analyses numériques corrélées, etc.). Idem pour ce qui concerne la production, il est important d'imaginer très en amont les moyens qui devront être mis en œuvre pour relever les challenges, voire paradoxes, liés aux enjeux de coûts, de qualité, de traçabilité et de pérennité.

Dans la suite de cet article, nous focaliserons le propos sur le marché aéronautique civil en sachant que nombre de sauts technologiques sont tirés par les besoins de défense et les opportunités qu'ils ouvrent du fait des financements étatiques, mais aussi des méthodes de certification spécifiques et des séries faibles. L'existence de démonstrateurs technologiques permet de renforcer la maturité des outils de justification et de certification qui seront utilisés pour le civil, et de compléter ou conforter les analyses de risques techniques et industriels. Le spatial, quant à lui, est un merveilleux booster pour la technologie et en particulier dans 
les domaines liés à l'allègement, la réduction des consommations électriques et l'adaptation à l'environnement spatial.

Il est important de noter que la prochaine génération d'avions ne verra le jour qu'à l'horizon 2025-2030, compte tenu des programmes de renouvellement en cours qui visent à répondre à un premier niveau de besoin d'évolution des compagnies aériennes en relation avec le coût de possession des aéronefs et leur impact sur l'environnement. Ils s'inscrivent également dans l'agenda stratégique fixé en 2000 par le conseil consultatif pour la recherche aéronautique en Europe, l'ACARE. Cet agenda vise à l'horizon 2020 que les développements technologiques pour les nouveaux avions tendent vers les objectifs suivants : la réduction de $50 \%$ des émissions des gaz à effet de serre, la réduction de $80 \%$ des émissions de NOx et la réduction de $50 \%$ du bruit perçu.

En 2011, un groupe de haut niveau représentant plusieurs secteurs d'activité (infrastructure, aéronefs, exploitation, carburants, recherche) a élaboré pour la commission européenne une nouvelle stratégie à long terme : Vision 2050. À cet horizon, les grands objectifs environnementaux fixés pour les nouveaux appareils sont : réduction de $\mathrm{CO}_{2}$ de $75 \%$ par passager-km (figure 2.1), réduction des $\mathrm{NOx}$ de $90 \%$, réduction du bruit perçu de $65 \%$ par rapport à une référence de l'année 2000. Ces objectifs seront parmi les lignes directrices qui constitueront les cahiers des charges des aéronefs et de l'ensemble des systèmes et des entités qui contribuent à leur fabrication, leur opération et leur maintenance.

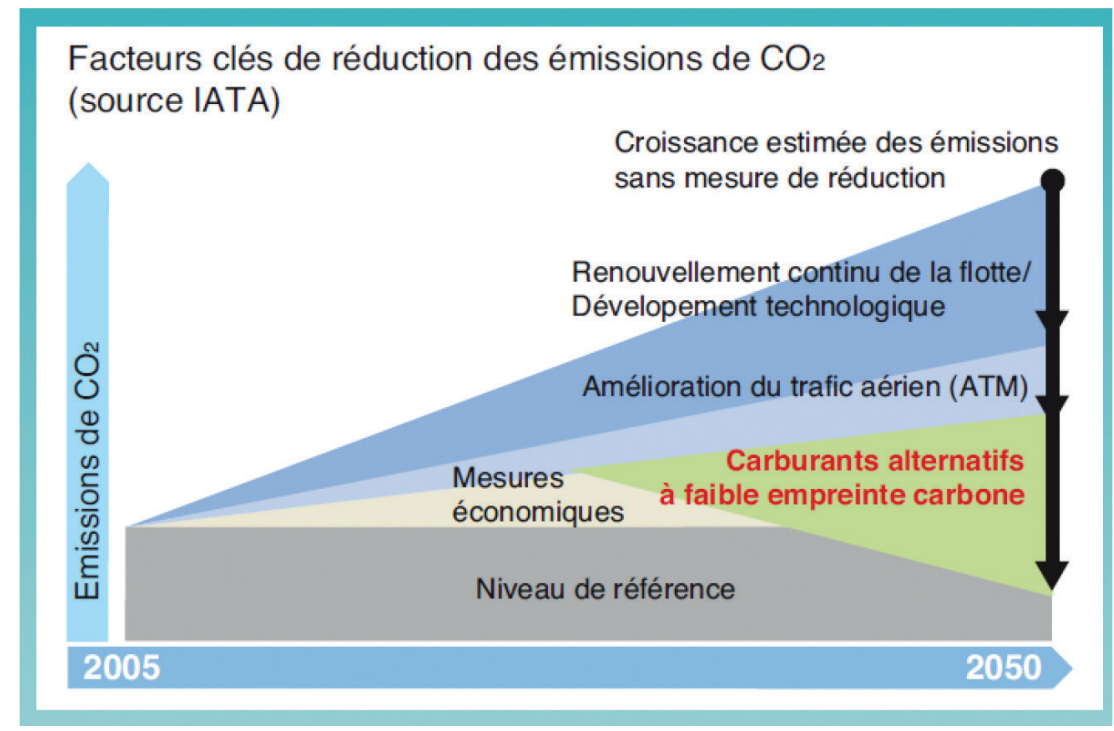

Figure 2.1. - Facteurs clés de réduction des émissions de $\mathrm{CO}_{2}$.

Il faut noter que l'atteinte de ces objectifs nécessitera la mobilisation de tous les talents du monde technique et scientifique car ils constituent une étape considérable à franchir. Cela venant s'ajouter aux progrès énormes déjà réalisés depuis 50 ans, avec, par exemple : la réduction d'un facteur proche de cinq de la consommation de kérosène (la consommation par passager d'un A380 est inférieure à 3 L/100 km), 
$20 \%$ de réduction du bruit à la source soit un facteur dix de l'amplitude sonore, réduction quasi totale des hydrocarbures imbrûlés et division par quatre des NOx [1].

Les principaux enjeux du domaine spatial seront de s'adapter aux évolutions du marché (nouveaux entrants et maîtrise des coûts tout en améliorant la flexibilité, la fiabilité et la disponibilité des produits). On considèrera en particulier la propulsion électrique des satellites et l'intégration des concepts de développement durable (ergols "verts », maîtrise des débris orbitaux et gestion de la fin de vie des satellites et étages supérieurs des lanceurs).

\subsubsection{Attentes vis-à-vis des partenaires-fournisseurs équipementiers-ensembliers}

Les principaux leviers d'innovation (figure 2.1) concernent bien évidemment la structure de l'aéronef et son système propulsif (moteur et nacelle), mais aussi tout ce qui touche au trafic aérien lui-même : temps de vol, altitude, trajectoire, optimisation de l'occupation de l'espace aérien.

Pour la structure (qui inclut l'ensemble constitué par la cellule et tous ses systèmes et équipements), il s'agit de trouver un équilibre optimum entre les forces qui s'exercent sur elle en réduisant le poids (allègement des structures, introduction de nouveaux matériaux métalliques, plastiques ou composites), en augmentant la portance pour certaines phases du vol, en réduisant la traînée et en améliorant l'utilisation de la poussée des moteurs par des installations innovantes. On doit également noter que l'optimisation de la masse de l'aéronef passe aussi par celle de l'ensemble de ses équipements et de ses systèmes.

Les turboréacteurs à double flux (figure 2.2) qui équipent la plupart des avions de ligne modernes sont apparus dans les années 1960. Comme tout moteur thermique, ils transforment l'énergie chimique contenue dans le carburant en une énergie mécanique propulsive. Le flux d'air " primaire » fortement comprimé et chauffé au travers du (ou des) compresseur(s), puis de la (ou des) chambre(s) de combustion, transforme l'énergie de la combustion en énergie mécanique grâce à la (ou aux) turbine(s) et à l'arbre (aux arbres). Cette énergie est ensuite utilisée pour comprimer le flux « secondaire », froid, pour créer l'essentiel de la poussée. Dans les turboréacteurs avec soufflantes, on ajoute un étage de diamètre bien supérieur au compresseur BP à l'avant de celui-ci. Cela permet d'obtenir du flux secondaire une poussée maximale. Un des paramètres essentiels d'optimisation de ce type de moteur est le taux de dilution, qui est le rapport du débit du flux secondaire au débit du flux primaire. Son optimisation va nécessiter de maîtriser l'aérodynamique des différents étages et leur interaction, mais aussi la combustion, la tenue mécanique de pièces soumises à des contraintes thermomécaniques extrêmes, tout cela en assurant fiabilité, sécurité, fabricabilité et capacité à une maintenance compatible avec les délais et les coûts objectifs.

Les équipements et les systèmes devront être encore plus légers et efficaces. De nombreux projets sont déjà en cours pour remplacer les vecteurs énergétiques classiques : air comprimé et fluides hydrauliques par le courant électrique afin d'obtenir une diminution significative de la consommation en carburant. C'est aussi l'occasion de mettre plus «d'intelligence » dans les équipements eux-mêmes, 
en permettant par exemple leur monitoring et leur pilotage fin in situ. Ce qui aura un effet direct sur la consommation, la masse et la fiabilité des équipements. Notons enfin que de nouveaux systèmes sont en cours de développement, qui viseront à assurer le déplacement au sol des avions sans avoir recours aux moteurs principaux. Ces systèmes (Electric Green Taxiing System, e-GTS) permettront des gains importants de consommation de carburant, mais contribueront aussi à la réduction du bruit sur le tarmac.
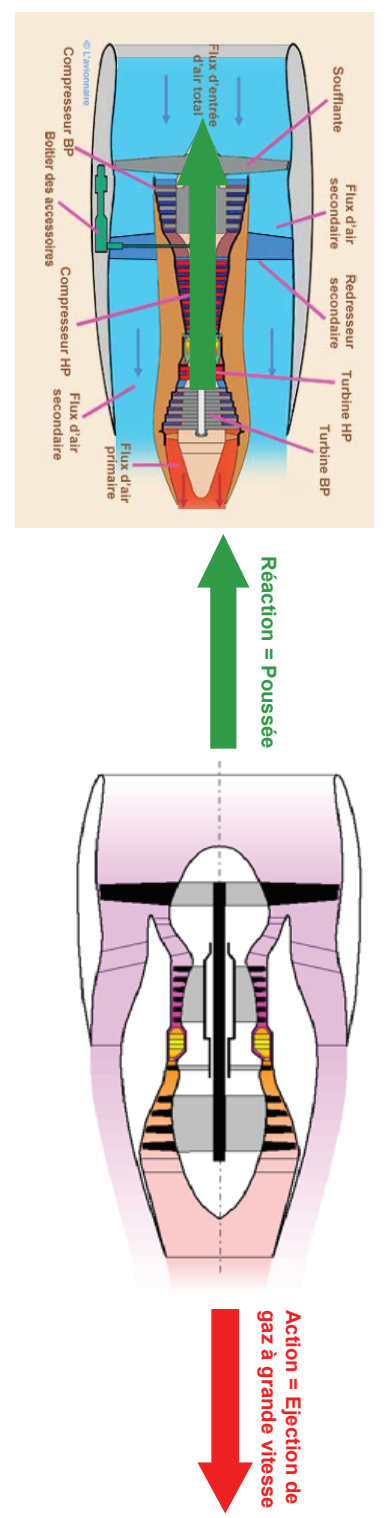

Figure 2.2. - Principe du turboréacteur à double flux équipé d'une soufflante [9]. 
Une approche toujours plus intégrée, très en amont, dans les travaux de recherche et développement de l'ensemble des acteurs permettra, en optimisant les compromis techniques nécessaires, de réduire l'impact environnemental des avions tout en protégeant l'économie de ce secteur structurant.

\subsubsection{Domaines clés de recherche et innovation}

L'atteinte des objectifs à moyen et long termes que le domaine transverse aéronautique et spatial s'est fixé en relation avec les enjeux économiques, environnementaux et sociétaux impose une mobilisation de l'ensemble de la communauté mécanicienne. En effet, les enjeux touchent tous les domaines depuis les matériaux (souvent le premier verrou technologique), au développement de concepts innovants permettant d'optimiser l'aérodynamique ou le design (au sens mécanicien du terme) des structures et des équipements à des fins de performances mécaniques ou acoustiques ou l'utilisation de nouvelles sources d'énergie, en passant par les aspects liés à l'ensemble du cycle de vie du produit (figure 2.3).

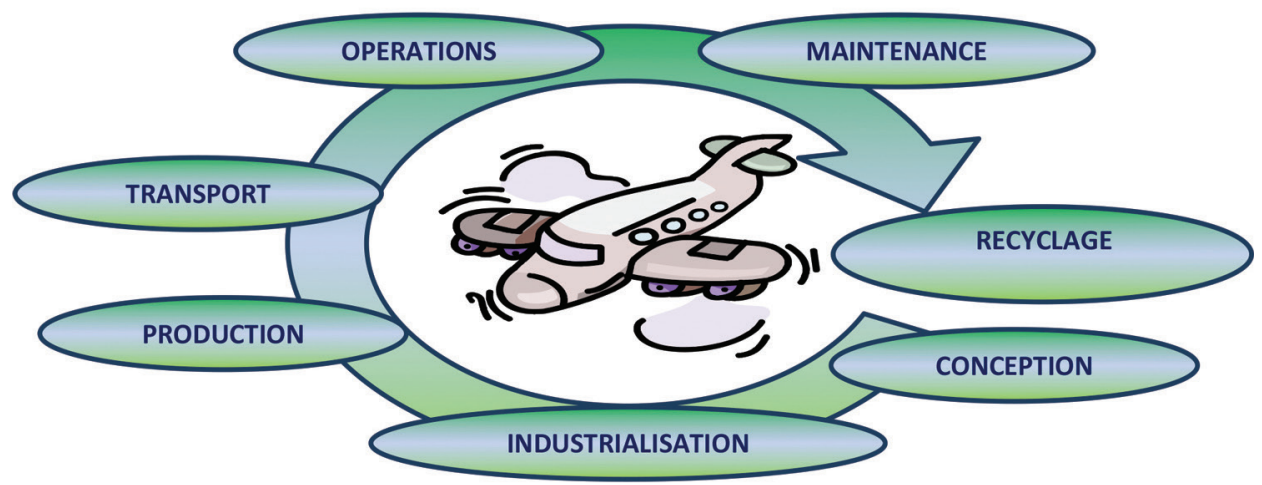

Figure 2.3. - Cycle de vie d'un produit.

Dans le domaine des matériaux, il va sans dire que de disposer de matériaux ayant des caractéristiques spécifiques (masse) optimales (mécaniques, thermiques, thermomécaniques, tribologiques, résistance aux chocs, corrosion, usure, compatibilités chimiques, etc.) est un « must». Mais la compréhension et la modélisation de leurs comportement et mode de ruine sont aussi indispensables pour permettre avec le moins d'essais possible de concevoir les systèmes en amont, puis de les justifier dans le cadre de leur certification. Il est donc important que très en amont, un effort particulier soit fait pour comprendre et modéliser leur comportement et leur mode de ruine sous sollicitations complexes et sévères. La capacité à prévoir très en amont le comportement mécanique et thermomécanique en relation avec le milieu dans lequel fonctionne le matériel considéré et/ou des moyens de mise en œuvre envisagés est capitale.

Dans le domaine de la modélisation (mécanique, thermomécanique, fluide...), les enjeux économiques sont énormes. Disposer de modèles prédictifs corrélés dans 
des domaines de validité connus et bien tracés (e.g. hautes températures, grandes vitesses de déformation, fatigue, vibrations, milieux corrosifs) est indispensable. Il faut néanmoins que les modèles mis en œuvre soient accessibles en termes de coûts et de moyens. Il faudra donc continuer à faire des efforts pour développer des outils numériques capables de s'adapter à la taille des projets et aux phases considérées (pré-développements, certification, revue de concept - PDR -, revue de figeage de concept - CDR -...). Mais au-delà des phases de conception, ces outils pourront être utilisés comme des outils de capitalisation de l'expérience des entreprises en les connectant à des outils de description des méthodes (guides ou chaînes de conception) ou en les liant aux résultats des essais avec lesquels ils auront été corrélés.

Dans le domaine des essais, il faut mettre en place des filières capables de développer et de maîtriser des moyens innovants qui permettront de disposer des éléments de corrélation et de preuves pour soutenir les justifications nécessaires à la certification des aéronefs, ou à la qualification des lanceurs et de leurs systèmes. Le développement de capteurs et des moyens associés de traitement des données ou des signaux pourrait également permettre de continuer à mettre plus « d'intelligence " au plus près de la fonction, et ainsi d'accéder à un monitoring approprié dont l'impact sur le «sur »dimensionnement inhérent à la nécessité de disposer de spectre de chargement générique pourrait être déterminant. On peut imaginer de même l'utilisation de capteurs capables d'optimiser une fonction de façon autonome. Cela pourrait permettre une réduction de consommation, de bruit ou de l'environnement induit sur la charge utile. Le développement de la mécatronique en milieu sévère est nécessaire pour soutenir ces innovations. En effet, il est important de rappeler qu'une des caractéristiques du secteur transverse aéronautique et spatial concerne les conditions extrêmes dans lesquelles doivent opérer régulièrement, voire en permanence, nos systèmes. En particulier, les conditions vibratoires, thermiques ou hydriques.

Dans le domaine de l'actuation, le passage de l'air comprimé ou de l'hydraulique vers le plus/tout électrique impose des sauts technologiques sans lesquels le poids et la consommation rendront impossible l'introduction de " l'avion tout électrique ». La communauté électromécanicienne doit se mobiliser en interaction très étroite avec les communautés électrotechnicienne, électronicienne et mécatronicienne pour mettre à disposition des équipementiers des systèmes puissants, légers et intelligents qui amélioreront la performance de leurs produits. Les micro- et nanotechnologies doivent faire l'objet d'une attention particulière afin d'atteindre les performances attendues. Attention! Il ne faut surtout pas que les communautés hydrauliciennes ou celles en relation avec l'air comprimé se démobilisent car les avions produits actuellement sont tous équipés de systèmes hydrauliques et pneumatiques, et voleront tous encore dans 30 à 40 ans. Il est donc important que ces produits puissent continuer à évoluer dans le cadre d'une innovation incrémentale pilotée.

Dans le domaine de la production et de l'ingénierie concourante, rien ne sert d'avoir fait une prouesse sur un ou des prototypes si, en parallèle, l'industrialisation n'a pas été préparée ni les schémas de production capables des performances de coûts et de qualité requis mis en place. Il est nécessaire de continuer à faire des efforts dans les différents domaines techniques de la production en parallèle des développements des nouveaux matériaux et des nouveaux concepts. Pour bien 
concevoir, nous avons besoin, très en amont, de développer les nouveaux concepts d'usinage, de formage, d'assemblage, de contrôle... pour disposer lors des phases de design et de dimensionnement des capacités, des coûts caractéristiques et des limites de chacune des techniques. Il est important de mettre en place les outils et les pratiques qui permettront de faire évoluer l'interface entre les concepteurs et les producteurs (ingénierie concourante) et ainsi concevoir dès le début des produits dont le coût de production cible et la fabricabilité sont bien projetés.

La communauté mécanicienne, dans son acception la plus large, est indispensable à la poursuite du développement du domaine aéronautique et spatial; nous en avons parlé tout au long de cet article. Mais elle doit continuer à coordonner ses efforts avec d'autres communautés indispensables : certaines semblent évidentes ou naturelles - comme celles des thermiciens ou des essais -, là où d'autres doivent le devenir - la production, l'électronique, l'électrotechnique, la mécatronique... Cela permettra de proposer les produits innovants, performants, fiables qu'attendent nos clients et à notre industrie de garder son rang mondial.

\subsubsection{Bibliographie}

[1] «Aéronautique, espace et environnement », GIFAS (Groupement des industries françaises aéronautiques et spatiales), brochure environnement.

[2] «Pourquoi le transport aérien a de beaux jours devant lui », Bruno Trévidic, Les Échos, 20 juin 2013.

[3] «Indicateur du trafic aérien mondial », Pierre Spacaro, traficaerien.canalblog.com, 12 décembre 2012.

[4] «Sur les perspectives d'évolution de l'aviation civile à l'horizon 2040 : préserver l'avance de la France et de l'Europe », rapport du Sénat 12-658, 15 septembre 2013.

[5] «Airbus optimiste pour l'essor du trafic aérien », Florentin Collomp, Le Figaro, 4 septembre 2012.

[6] IATA communiqué $\mathrm{n}^{\circ} 25,1^{\mathrm{er}}$ mai 2013.

[7] «IATA : trafic mondial en hausse de 5,9\% en mars », www.air-Journal.fr.

[8] «Quel avenir pour l'industrie aéronautique et spatiale européenne ? », dossier $\mathrm{n}^{\circ} 36$ de l'Académie de l'air et de l'espace, 2013.

[9] http://www.lavionnaire.fr/MotorTurboReact.php.

[10] ASD-Eurospace, Facts E Figures, 16th Edition, June 2012.

[11] ASD-Eurospace, "State of the European Space Industry", Web Release, June 2013.

\subsection{L'énergie}

Le vaste champ des consommations d'énergie et des formes, très diverses, de production d'énergie est l'un des champs privilégiés de la mécanique, dans nombre de ses dimensions : mécanique des mouvements, mécanique des solides et des structures, mécanique des fluides, matériaux, souvent en interaction avec les grandes disciplines que sont la chimie (voir le rôle considérable de la combustion 
dans les procédés de production d'énergie) et la physique, au sens classique du terme, une acception selon laquelle on oublie souvent que la mécanique est une branche de la physique - la première d'ailleurs où l'on a fait du quantitatif - en y intégrant l'astronomie.

On peut considérer l'ensemble des secteurs de production, de diffusion et d'usage de l'énergie en faisant référence au «bouquet énergétique » et en distinguant les énergies fossiles, l'énergie nucléaire qui, quelles qu'en soient les évolutions, relève d'une analyse particulière, les énergies renouvelables traditionnelles et toutes les formes d'énergie renouvelable qui sont actuellement en plein développement relatif.

\subsubsection{Les énergies fossiles}

Les plus anciens combustibles fossiles - la tourbe, le lignite et, bien sûr, le charbon ne voient pas actuellement leur évolution marquée par des innovations techniques majeures. Ce qui ne signifie pas que, comme toutes les technologies matures, ils ne connaissent pas de progrès. L'une des solutions - souvent évoquée, peu mise en œuvre - consisterait, par exemple, à développer des exploitations « in situ », pour des productions de méthane, en utilisant des puits pour accéder aux gisements, au sein même desquels se développeraient les réactions de transformation. Un tel « rêve » présenterait l'intérêt de permettre le traitement de gisements profonds et souvent d'épargner des vies humaines (le charbon est considéré comme la plus «meurtrière» des sources d'énergie).

\subsubsection{La filière Oil and Gas}

\subsubsection{Importance macroéconomique du secteur}

L'industrie parapétrolière et paragazière regroupe l'ensemble des sociétés de services pétroliers et gaziers qui réalisent les travaux d'étude et de construction nécessaires à l'exploitation des gisements d'hydrocarbures pour le compte des compagnies. L'expertise de ces sociétés couvre tout le champ de la filière hydrocarbures (exploration, production, développement, logistique, raffinage, distribution) avec des activités très diverses allant de la réalisation d'études sismiques à la construction de plates-formes, d'unités de raffinage ou de traitement de gaz, en passant par la conception et la mise au point d'équipements spécifiques.

Le chiffre d'affaires total pour 2012 est estimé à 35 milliards d'euros générés depuis la France par environ 400 sociétés. La part prise par les travaux en mer a atteint environ $40 \%$ du montant global estimé. L'effectif total du secteur est estimé à 60000 personnes en France (hors secteurs de la distribution et du commerce).

Environ 350 PME réalisant un CA inférieur à 200 millions d'euros contribuent activement au chiffre d'affaires global pour près de 7 milliards d'euros (18\%), tandis que 35 sociétés de plus de 200 millions d'euros de CA se partagent le solde, soit 28 milliards d'euros (82\%).

L'activité parapétrolière est l'activité unique ou majoritaire de près de 200 entreprises, la moitié restante offrant également des services à d'autres industries. 
L'implantation des sièges sociaux sur le territoire national privilégie l'Île-deFrance mais s'avère notable dans les régions Rhône-Alpes et Provence - Alpes Côte d'Azur. Tournées vers l'exportation, les principales entreprises françaises sont présentes ou représentées dans 70 pays étrangers. Leur expertise est internationalement reconnue.

Selon une enquête réalisée en 2013 par le GEP-AFTP auprès d'une centaine d'entreprises, la part à l'exportation du chiffre d'affaires parapétrolier est en moyenne de $56 \%$ pour l'ensemble de l'échantillon, mais atteint plus de $70 \%$ dans le cas de grands groupes dont le chiffre d'affaires total est supérieur à 200 millions d'euros.

La majorité des entreprises anticipaient pour 2013 une hausse du chiffre d'affaires parapétrolier ainsi que de la part à l'exportation. En termes d'emploi, $68 \%$ des entreprises interrogées ont recruté un total de plus de 2000 personnes en 2012 et $65 \%$ avaient l'intention de recruter de nouveau en 2013. Toutefois, $63 \%$ éprouvaient des difficultés à trouver des candidats répondant à leurs besoins.

L'effort en matière de recherche et développement est soutenu puisque $27 \%$ des sociétés interrogées, en particulier les PME/PMI, affirmaient dépenser plus de $5 \%$ de leur chiffre d'affaires à ce poste.

Les tendances dégagées par l'enquête donnent l'image d'un secteur industriel dynamique dont l'activité est en croissance. La filière parapétrolière et paragazière française est donc constituée de quelques grands ensembliers de renommée internationale et d'un grand nombre de PME/PMI. Ces dernières concourent à apporter des solutions innovantes sur la base d'un important effort de R\&D.

\subsubsection{Tendances, enjeux et défis du secteur à l'horizon 2020}

Les principaux enjeux et les objectifs du secteur à l'horizon 2020 sont :

- baisser les coûts d'exploration et de production ;

- améliorer les technologies existantes et développer de nouvelles technologies.

Les sujets principaux de ces enjeux sont:

$\checkmark$ les sables bitumineux, huiles et gaz non conventionnels ;

$\checkmark$ les fluides complexes : gaz acides, HP/HT $\left(\mathrm{T}>180{ }^{\circ} \mathrm{C}, \mathrm{P} \geq 2000\right.$ bar $)$, huiles visqueuses ;

$\checkmark$ la récupération assistée (EOR) ;

$\checkmark$ les grandes profondeurs d'eau (entre 300 et $4000 \mathrm{~m}$ ) ;

$\checkmark$ les zones arctiques.

Les deux derniers items constituent les plus grands défis du secteur.

Au début des années 2000, les dépenses pour le «deep offshore » s'élevaient à 7 milliards de dollars. Le marché a énormément progressé pour atteindre un niveau de plus de 30 milliards de dollars en 2008. Après la crise financière, les opérateurs ont réduit leurs investissements. Depuis 2011, la croissance est retrouvée ; c'est ainsi qu'en 2014, la dépense d'investissements est estimée à 45 milliards de dollars. Suivant les opérateurs, les dépenses devraient croître à un taux annuel de $15 \%$ pour atteindre 115 milliards de dollars en 2020. 


\subsubsection{Attentes vis-à-vis des partenaires-fournisseurs équipementiers-ensembliers}

La filière parapétrolière et paragazière est très vaste et en constante nécessité d'innovation. Les besoins sont donc importants dans des domaines variés comme: l'exploration et la production, la sécurité, le transport et le stockage, sans oublier l'environnement et le «local content », c'est-à-dire la volonté des pays d'employer du personnel et de fabriquer localement.

\subsubsection{Domaines clés de recherche et innovation}

Au sein du GEP-AFTP, il existe un programme de financement de projets de R\&D financés par des sponsors privés : Total, GDF Suez, Technip, Saipem, Subsea 7, DORIS, CGG, Entrepose, Schlumberger et Geoservices. Ces sponsors proposent des pistes de recherche et d'innovation dans le domaine de l'exploration de production des hydrocarbures, et à destination de PME et sociétés innovantes.

Ces sponsors ont présenté des sujets tels que :

$\checkmark$ fluides complexes ;

$\checkmark$ matériaux de tout type : polymères, métaux, ciments, performants en conditions extrêmes ;

$\checkmark$ nouveaux capteurs et leur électronique associée dans des conditions extrêmes ;

$\checkmark$ robotisation, automatisation ;

$\checkmark$ ergonomie opérateur, virtual training;

$\checkmark$ gestion de gros volumes de données;

$\checkmark$ connaissance des réservoirs pétroliers ;

$\checkmark$ forage/production : détection des puits proches, analyse de la boue, et gestion des eaux de production ;

$\checkmark$ forage/production en offshore très profond : déploiement des conduites au fond de la mer, nouveaux risers, équipements au fond, traitement des effluents ;

$\checkmark$ GNL (gaz naturel liquéfié) offshore: pipes cryogéniques, transport, stockage ;

$\checkmark$ environnement : traitement des fuites de pétrole accidentelles, effet des émissions acoustiques sur le milieu marin.

Reste à considérer le domaine actuellement très discuté des gaz de schiste. Au-delà des débats, en France, sur l'existence de réserves importantes et sur la gestion des eaux usées lorsque la fracturation des sols est faite en ayant recours à l'eau, les principaux problèmes mécaniques sont liés aux conditions de réalisation d'un réseau de fissures efficace, à sa tenue en fonction du temps... Les réflexions sur des agents de fracturation autres que l'eau sont en cours, aux frontières de la chimie et de la mécanique. 


\subsubsection{L'énergie nucléaire}

La production d'énergie, presque exclusivement sous forme d'électricité, par voie nucléaire, a commencé en 1957. Après un développement régulier et relativement important, s'appuyant sur plusieurs types de réacteurs, la situation s'est stabilisée autour de modèles à eau légère. La production d'électricité dans le monde, antérieurement à la catastrophe de Fukushima, marque le pas. Dans ce domaine, on distingue bien trois catégories de composants : ceux qui relèvent du " consommable ", comme le combustible, ceux qui sont remplaçables, à l'occasion par exemple de visites techniques, et ceux qui ne sont pas remplaçables, au premier rang desquels figure bien sûr la cuve.

\subsubsection{La construction nucléaire}

On ne traite dans cet article que le nucléaire civil. Sont couverts non seulement la construction, mais aussi l'exploitation et la maintenance.

\subsection{Importance macroéconomique du secteur}

Le secteur de l'énergie nucléaire est très développé en France ; il est l'un des premiers dans le monde.

Sans parler de la partie militaire, la partie civile comporte principalement :

1. l'exploitation par EDF de 56 réacteurs qui assurent près de $80 \%$ de la production française d'électricité ;

2. une industrie complète, avec :

- pour les réacteurs, la conception et la construction de réacteurs électrogènes et l'ensemble des composants et matériaux spéciaux,

- pour le combustible, un cycle passant par la mine, la fabrication du combustible et son retraitement ;

3. un domaine de la recherche très développé, avec des installations au premier rang mondial.

La France exporte des réacteurs depuis près de trente ans et, désormais, les industries de premier rang ainsi que leurs sous-traitants sont reconnus et exportent aussi leurs produits et savoir-faire.

Le nucléaire représente :

- 125000 emplois directs, soit $4 \%$ de l'emploi industriel en France ;

- 410000 emplois totaux (directs, indirects, induits), soit $2 \%$ de l'emploi en France ;

- trois fois plus d'emplois créés en France pour chaque euro investi que les autres filières de production d'électricité ;

- une valeur ajoutée totale de 33 milliards d'euros en France, soit une contribution totale équivalente à $2 \%$ du PIB français. 


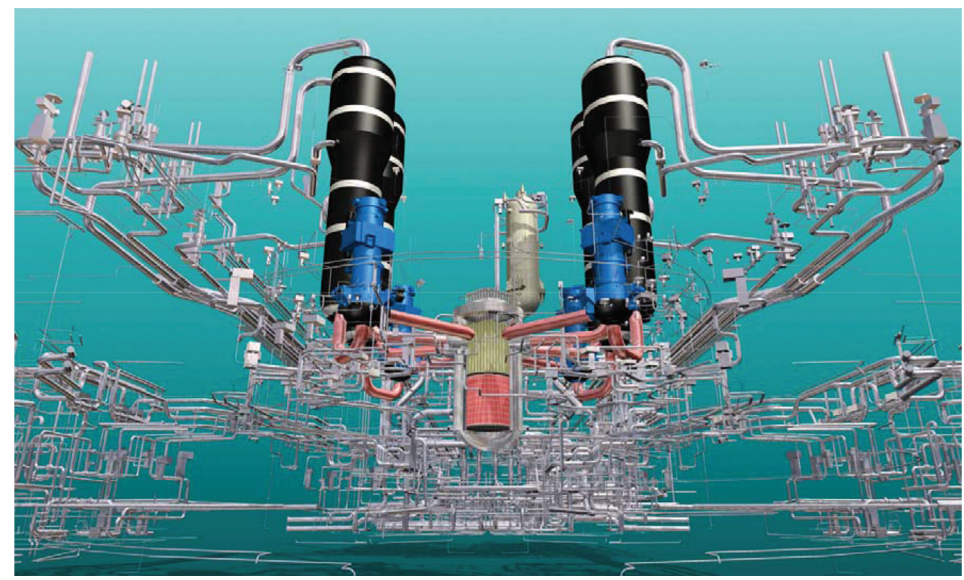

FIGURE 2.4. - Image numérique des circuits primaire et secondaire et des auxiliaires de l'EPR.

\subsection{Tendances, enjeux et défis du secteur à l'horizon 2020}

La production d'électricité restera majoritairement nucléaire en France car les alternatives (éoliennes, solaires) ne pourront pas à cet horizon être installées à un niveau suffisant de capacité et à un coût d'installation compétitif.

Dans le monde, de nombreux pays s'appuient sur le nucléaire, ce qui donne des opportunités très importantes pour les industries françaises.

Des chantiers de nouveaux réacteurs existent actuellement en France, en Finlande, en Chine, aux États-Unis et au Brésil. On attend prochainement la construction de nouveaux réacteurs anglais.

Il apparaît que les gaz de schiste deviennent le concurrent le plus sévère au nucléaire.

Si l'on peut dire que les États-Unis ont perdu leur statut de leader mondial, qui maintenant peut être revendiqué par la France, on peut supposer que dans une génération, la Chine sera devant nous si elle continue au rythme actuel. C'est un enjeu stratégique de se positionner par rapport à ce grand pays.

\subsection{Attentes vis-à-vis des partenaires-fournisseurs équipementiers-ensembliers}

Les clients sont les « utilities », producteurs d'électricité exploitants nucléaires. En France, il s'agit d'EDF.

AREVA est le constructeur européen de centrales (basé en France, filiales en Allemagne et aux États-Unis).

S'il est vrai qu'EDF et AREVA sont les acteurs majeurs de la filière, le volume des études ou fabrications sous-traitées est très important. L'enjeu est, tant en France que dans les autres pays du monde, de retrouver des fournisseurs qualifiés. La construction nucléaire a été ralentie pendant deux décennies. Certes, on peut se réjouir d'un regain de nouveaux projets, mais il est difficile de retrouver tout 
le tissu industriel et la "culture » de qualité nucléaire. Alors que les petites sociétés cherchaient d'autres débouchés, on a observé dans le même temps un durcissement progressif des règles imposées par les autorités de sûreté, qui risquent d'éloigner de plus en plus les fabrications nucléaires des fabrications courantes de l'industrie. Il y a donc un double défi :

- ne pas différencier inutilement les niveaux d'exigences nucléaires du reste de l'industrie,

- recréer des compétences «nucléaires » dans l'industrie dans son ensemble.

Ce sont des défis mondiaux et si les entreprises françaises les surmontent, elles pourront prétendre aux marchés exports.

\subsection{Domaines clés de recherche et innovation}

La mécanique est présente dans les domaines suivants :

- mécanique des fluides :

o simple phase,

o couplage avec les réactions neutroniques,

o couplage avec le comportement des systèmes ;

- échanges de chaleur et "thermo-hydraulique" :

o double phase (par exemple eau-vapeur) ;

- dynamique :

o séisme,

o simulation d'accidents (graves),

o chute d'avion,

o vibrations ;

- thermomécanique :

o comportement non linéaire,

o endommagement (fatigue) ;

- rupture ;

- effets de vieillissement des matériaux :

o irradiation,

o corrosion,

o usure ;

- simulation multi-physique :

o interactions fluides/structures ;

- procédés de fabrication : interaction mécanique/métallurgie :

o solidification des lingots,

o forgeage, 
o soudage,

o usinage,

o traitements thermiques;

- «vieillissement» en service (effets décrits ci-dessus).

Les problèmes sont abordés par simulation et essais dans des centres dédiés, principalement au CEA, chez EDF et chez AREVA.

Les logiciels utilisés sont soit des logiciels "généraux » du commerce (éléments finis, mécanique des fluides par exemple), français ou non (la France occupant le premier rang mondial), soit des logiciels «spécialisés »spécifiques de la filière nucléaire, dont là encore, la France est leader mondial. On constate que les logiciels commerciaux prennent progressivement de l'importance dans des domaines non exclusivement nucléaires : ayant une base de marché plus grande, ils peuvent se développer avec des équipes conséquentes. L'effort pour les logiciels spécialisés, qui était très important dans les années 1980, se réduit mondialement. Cependant, en France, il demeure très significatif dans notre décennie. La Corée apparaît dans ce domaine, mais pas la Chine.

On n'oubliera pas les idées de développement de ce que l'on appelle la génération IV, surgénératrice, pour laquelle le problème principal est lié, pour l'instant, à l'utilisation du sodium, un problème qui n'est pas seulement mécanique.

En ce qui concerne le futur, relativement lointain, ce n'est pas avant la fin de la seconde moitié de ce siècle que la fusion contrôlée pourrait, peut-être, apporter sa contribution à la demande en énergie. Sur ce thème, de nombreux problèmes de physique se posent, mais l'essentiel se situe au niveau des matériaux, qui doivent pouvoir résister à l'érosion du plasma et aux flux de neutrons. Parmi les deux solutions possibles, c'est la voie par confinement magnétique qui mobilise actuellement les chercheurs (différents tokamaks, le JET, ITER...).

\subsubsection{Les énergies renouvelables traditionnelles}

Il ne faut pas perdre de vue que les énergies renouvelables furent les premières formes d'énergie utilisées, dans le but de produire du feu, puis, via divers processus, de l'électricité. Dans certaines zones, où la consommation d'énergie par habitant est modeste, la part des énergies renouvelables dans le bouquet énergétique est encore très importante, voire majoritaire. C'est le cas, par exemple, du continent africain dans son ensemble où, en moyenne, la part des énergies renouvelables avoisine les $75 \%$ de toutes les formes d'énergie utilisées. Par ses liens historiques et culturels avec l'Afrique, la France peut jouer un rôle important dans l'évolution du mix énergétique sur ce continent.

L'hydraulique est la forme la plus anciennement utilisée, avec le feu et l'énergie éolienne, des énergies renouvelables. L'exploitation en France de cette forme d'énergie, "la houille blanche », a très tôt été fortement développée. Les problèmes mécaniques principaux de ce type de production restent liés aux barrages, avec, d'une part, des problèmes de sécurité liés tout particulièrement aux barrages-voûtes et à leur accroche sur les terrains encaissants, et, d'autre part, au vieillissement du parc, un problème de plus en plus rencontré actuellement. 
Le second secteur d'importance est, bien entendu, celui de la biomasse, ou plus exactement des biomasses. Qu'il s'agisse de la récolte, du traitement (combustion, méthanisation...), de la transformation (les biocarburants par exemple), voire, demain, des démarches impliquant les microalgues, il y a dans ce secteur une croissance importante de produits nouveaux intéressants ou de procédés adaptés qui se développent, parfois très fortement, à une vitesse entraînant à l'évidence la participation d'industriels, relevant souvent du secteur mécanique, pas toujours de très grande taille, de manière importante (on peut prendre en exemple le développement des chaudières à gaz à condensation, ou celui spectaculaire des «pellets» pour divers poêles et chaudières).

\subsubsection{Autres formes d'énergie renouvelable en pleine expansion}

Les crises énergétiques, l'accroissement des populations, le souci de chacun d'un minimum vital ont constitué des facteurs stimulants, et ce depuis le choc pétrolier de 1973, mais plus encore avec les crises récentes, pour une très grande variété de sources d'énergie renouvelable, parfois assez inédites par rapport à ce qui se faisait il y a moins de dix ans.

Digne successeur d'une activité ancienne, celle des moulins à vent, l'énergie éolienne connaît depuis une dizaine d'années, partout dans le monde, un développement considérable. Actuellement, ce ne sont pas moins d'une trentaine de firmes industrielles qui produisent, de par le monde, les éoliennes «standard", d'une puissance de 2 à $5 \mathrm{MW}$ en moyenne. Des projets de plus grande puissance, jusqu'à $8 \mathrm{MW}$, sont en cours et, qu'il s'agisse des machines elles-mêmes, de l'amélioration de leur rendement, de leur mise en place ou de leur interaction avec les courants d'air locaux, les sujets de réflexion et de recherche ne manquent pas. Fin 2013, la puissance totale des éoliennes en fonction était, dans le monde, d'un peu plus de $300 \mathrm{GW}$, ce qui, en prenant en compte un taux de charge de $25 \%$ en moyenne, correspond à près d'une centaine de "tranches ", nucléaires ou à charbon, de 1000 MW environ (avec un taux de charge de $80 \%$ ). Le taux de croissance annuel est nettement « à deux chiffres ».

On peut évoquer ici, car c'est bien souvent avec l'éolien qu'on les évoque, avec ses intermittences naturelles, les problèmes et techniques de stockage de l'énergie, de l'électricité en particulier. En dehors des stockages gravitaires qui impliquent l'existence de deux « réservoirs » d'eau à des altitudes différentes, ce qui correspond à des géographies particulières (la Suisse est bien évidemment un bon exemple pour la mise en œuvre, effective, de cette technique), d'autres techniques de stockage sont à l'étude, comme dans le domaine du photovoltaïque. Nous y reviendrons. Le problème de la gestion fine des réseaux, compte tenu du développement des sources intermittentes, avec la problématique dite des «smart grids », est important mais ne relève que très partiellement des compétences des industries mécaniques.

S'agissant de l'usage potentiel des éléments naturels pour la production d'électricité, on doit noter le développement de nombreux projets d'exploitation des courants marins, des marées, de la houle... Le développement des hydroliennes est un 
champ actuellement en plein essor. Sur tous ces sujets, la mécanique en général, qu'il s'agisse de l'étude des systèmes ou des matériaux, est bien sûr mise à contribution.

C'est aussi le cas pour ce que l'on appelle la «thermodynamique solaire " ou le «solaire à concentration", pour lesquels divers dispositifs (champs de miroirs cylindro-paraboliques, tours à concentration, miroirs paraboliques, miroirs de Fresnel) sont testés un peu partout dans le monde, en grandeur nature. Il existe des installations fonctionnant depuis plus de vingt ans, avec des productions de l'ordre de la centaine de mégawatts ; toutes sont structurées de manière à stocker de l'énergie, sous forme de sels fondus par exemple, permettant de compenser les périodes sans soleil, avec éventuellement le couplage avec des turbines à gaz.

Enfin, on ne saurait oublier les systèmes photovoltaïques qui, avec les « fermes éoliennes ", constituent une source, non négligeable, de production électrique. Leur développement se fait actuellement à un rythme toujours plus important (une croissance également "à deux chiffres », comme pour l'éolien) : fin 2012, la puissance installée dans le monde était supérieure à 100 GW crête, soit avec un taux de charge moyen estimé à $12 \%$ environ, l'équivalent d'une quinzaine de «tranches » de centrale nucléaire. Mais le photovoltaïque concerne peu les mécaniciens.

Les évolutions des modes de production de l'électricité, avec la montée en puissance de formes de plus en plus intermittentes, éoliennes et photovoltaïques en particulier, mettent l'accent sur des vecteurs d'énergie intermédiaires qui pourraient jouer un rôle tout à la fois dans le domaine du stockage de l'électricité et dans celui de l'usage direct de ces vecteurs énergétiques. C'est le cas, en particulier, de l'hydrogène.

Si l'emploi de l'hydrogène, comme fluide industriel, est courant depuis bien longtemps avec plusieurs centaines de kilomètres de « pipes » de par le monde, en Europe notamment, la mise au point de systèmes de stockage intégrés à des dispositifs de production d'électricité fait l'objet d'expériences et de projets pilotes variés. Au-delà de cette voie qui repose toujours sur une production solaire d'électricité, suivie d'une phase d'électrolyse de l'eau, les différents modes de stockage de l'hydrogène, en vue d'applications dans les transports, font et feront à court terme l'objet de nombreux travaux : il peut s'agir de stockage en masse sous forme liquide ou gazeuse, ou de stockage sous forme adsorbée, sur des solides divisés de compositions chimiques variées.

\subsubsection{Des politiques d'économie d'énergie}

Ont été évoquées ici les différentes formes de production d'énergie, en ne perdant pas de vue la vision «vecteurs énergétiques». Il ne fait aucun doute que l'approche "consommation " plutôt que "production "se révélera féconde pour la mise en place du principal, les stratégies et tactiques au service d'une vision essentielle, la réduction des consommations d'énergie dans maints secteurs par l'utilisation rationnelle de cette énergie.

Le secteur de l'énergie est, avec la gestion de l'eau et des matières premières (y compris la gestion des déchets), l'un des grands problèmes des prochaines décennies, à l'échelle mondiale, auquel il convient d'adjoindre les activités relevant de l'informatique et de la communication, secteur qui demande une attention toute 
particulière. La population mondiale a considérablement augmenté ces dernières décennies, sans que les inégalités soient pour autant significativement réduites.

Le secteur de l'énergie est donc bien un secteur où nombre des concepts qui ont fortement émergé ces dernières années vont prendre tout leur sens, dans le cadre de ce que l'on désigne parfois sous le nom de «transition énergétique »: analyse des cycles de vie, écologie industrielle, généralisation des approches systémiques, écoconception... Il est évident qu'il nécessitera des mécaniciens.

La mécanique française a permis au cours des siècles passés de fournir au pays l'énergie dont il avait besoin. Dans le futur, et aussi dans le contexte des débats actuels sur l'énergie, il est évident que les mécaniciens doivent encore jouer un rôle essentiel.

\subsection{L'industrie ferroviaire}

\subsubsection{Importance macroéconomique du secteur}

L'histoire du transport par chemin de fer remonte aux débuts de l'ère industrielle. L'essor de ce mode de transport se situe vers les années 1850 avec les tramways tirés d'abord par des chevaux à Londres, Berlin, Paris, Milan, et un boom ferroviaire à la même époque avec les locomotives à vapeur. Par ailleurs, c'est à la fin du $\mathrm{XIX}^{\mathrm{e}}$ siècle que le métro est apparu à Londres, puis à Budapest et à Paris. Ce mode de transport a dominé pendant près d'un siècle avant d'être supplanté, depuis la fin de la Seconde Guerre mondiale, par le transport routier automobile.

Après la machine à vapeur, c'est la traction électrique et le moteur Diesel qui révolutionnent le moyen de propulsion, et c'est vers 1930 que les réseaux ferrés se généralisent en France. À partir des années 1980, les trains à très grande vitesse (Shinkansen au Japon, TGV en France, ICE en Allemagne) révolutionnent l'image de ce mode de transport qui reprend des parts de marché à l'aéronautique sur les distances inférieures à $1000 \mathrm{~km}$.

Aujourd'hui, le marché mondial du matériel ferroviaire roulant est estimé à 39,5 milliards d'euros et connaît actuellement une croissance annuelle moyenne de $3,2 \%$.

En France, ce secteur est constitué par environ 250 entreprises, dont une quarantaine d'équipementiers de rang 1 réalisant un chiffre d'affaires de l'ordre de plus de 4 milliards d'euros pour un effectif d'environ 16000 salariés en France. Alstom Transport est l'ensemblier dominant et représente les 2/3 de l'effectif et du chiffre d'affaires du secteur. Le second ensemblier étant Bombardier Transport, avec environ 1700 salariés en France. Les autres entreprises de ce secteur sont des équipementiers de premier rang, dont le principal est Faiveley Transport avec environ 1000 salariés, des équipementiers de deuxième rang (comme Mersen à Amiens ou Stratiforme Industries à Bersée) et des sous-traitants.

Le domaine de l'infrastructure ferroviaire compte également quelques grandes entreprises françaises dans les domaines du génie civil, des équipements de voie, comme de la signalisation. Il représente un marché mondial de 45,7 milliards d'euros - de 4,4 milliards d'euros en France - pour 22000 emplois. 
Parmi les principaux acteurs européens, on peut citer : Thales ou Ansaldo STS pour le domaine de la signalisation, Egis Rail, Vossloh Cogifer, Tata Steel, Colas Rail ou encore Bouygues pour la construction de voies.

Les sociétés d'ingénierie comme Systra bénéficient d'une renommée mondiale pour la conception de systèmes complets de voie.

Les exploitants, bien que n'étant pas dans ce secteur industriel, tels que la SNCF ou la RATP, disposent de filiales, regroupant près de 100000 salariés, qui participent au progrès du secteur par la réalisation de travaux de maintenance et de développement de l'infrastructure, et leurs services d'ingénierie.

La SNCF et le RFF sont également associés aux principaux industriels du secteur ferroviaire, dans l'institut de recherche technologique (IRT) Railenium, qui rassemble les meilleurs chercheurs français dans les domaines d'expertises liés au ferroviaire.

\subsubsection{Tendances, enjeux et défis du secteur à l'horizon 2020}

Le secteur est confronté à une double tendance.

- D'une part, un développement du transport de passager depuis l'avènement du TGV, qui devrait se poursuivre avec des trains allant encore plus vite, mais qui dépend essentiellement de la construction de nouvelles infrastructures par les États dans lesquels ces réseaux ferrés se développeront, les infrastructures existantes ne pouvant supporter des trains à grande vitesse. Également au niveau du transport de passagers, le renouvellement du transport urbain et régional, notamment les tramways, qui implique aussi des infrastructures spécifiques dont l'investissement dépend de la politique des agglomérations et régions.

Il est clair dans ce contexte que les enjeux, tant environnementaux que socio-économiques, renforcent l'attractivité du transport ferroviaire pour lequel les constructeurs, les exploitants du matériel roulant et les gestionnaires de l'infrastructure sont soumis à des contraintes de plus en plus sévères. Il faut aller plus vite, à des prix compétitifs, tout en assurant un confort aux usagers en minimisant autant que possible l'impact sur les riverains (bruit et vibrations).

- D’autre part, il s'agit de répondre aux enjeux sécuritaires ; en particulier, une partie du réseau ferroviaire est vieillissante, les coûts d'entretien et de réparation ne cessent d'augmenter, la fiabilité se dégrade. Pour minimiser ce processus à la fois coûteux et chronophage, tous les acteurs s'accordent à dire que la voie ferrée du futur doit faire l'objet d'une analyse globale prenant en compte l'ensemble des éléments qui la constituent et la sollicitent, depuis le véhicule jusqu'au sous-sol (Railway Track Sciences and Engineering - Ballast : Issues and Challenges, Paris, décembre 2013), tout en considérant qu'il faut développer en parallèle de nouveaux systèmes de surveillance et de détection des défauts, ainsi qu'une signalisation apportant une aide intelligente pour répondre à ces défis de sécurité. 
Enfin, en ce qui concerne plus spécifiquement le fret ferroviaire, la construction de wagons de marchandises est en forte régression. Après un début de $\mathrm{XXI}^{\mathrm{e}}$ siècle marqué par une forte demande de matériel pour faire face à la croissance économique ressentie dans toute l'Europe, le secteur s'est doté de fortes capacités de production qui ont, peu à peu, saturé la demande. Alors que le fret ferroviaire génère plus de trafics dans d'autres pays européens, il semble poursuivre son déclin en France.

Cependant, un enjeu majeur demeure sur le long terme afin de faire passer le transport de marchandises des routes au rail, dans le but de réduire les embouteillages sur les routes et les émissions de $\mathrm{CO}_{2}$. Mais la libéralisation totale des services de fret ferroviaire début 2007 en Europe n'a pas encore conduit à des résultats marquants.

\subsubsection{Attentes vis-à-vis des partenaires-fournisseurs équipementiers-ensembliers}

Les principaux leviers d'innovation de ce secteur se situent dans la recherche d'une économie durable, les trains, métros, tramways apportant un mode de transport qui réduit l'impact environnemental du transport terrestre dans l'optique d'une optimisation de la gestion de l'énergie et d'une réduction de l'empreinte carbone. Mais il ne faut pas pour autant négliger les autres modes de transport et l'extension de la comodalité, qui sera une nécessité pour une attractivité et une accessibilité des transports, et devrait conduire au développement de nouveaux modèles socio-économiques de l'innovation.

Actuellement, les exploitants de ce secteur sont très exigeants sur la fiabilité et la qualité des matériels pour assurer la sécurité des personnes et des opérateurs, et imposent des cahiers des charges contraignants qui se basent sur des réglementations et des normes. Ce qui conduit à des coûts et des délais importants de mise sur le réseau freinant le développement de l'innovation.

Les partenaires et fournisseurs des constructeurs de matériels roulants et des infrastructures auront, dans ce contexte, à travailler plus étroitement ensemble pour répondre aux défis d'un secteur en plein renouveau, comme l'attestent les nombreux projets collaboratifs du pôle de compétitivité I-Trans. C'est cette volonté qui a permis la création de l'IRT Railenium.

\subsubsection{Domaines clés de recherche et innovation}

Comme dans les autres secteurs du transport, la recherche d'allègement est une préoccupation majeure, qui a pour objectifs de réduire la consommation d'énergie et l'impact en $\mathrm{CO}_{2}$, mais aussi de diminuer la pénibilité des tâches des personnels qui manipulent des pièces parfois lourdes, comme par exemple un tendeur d'attelage. Il s'agit d'étudier des pièces mécaniques fabriquées dans des matériaux légers et résistants : composites, titane, mais aussi aciers à haute résistance en réduisant la matière utilisée tout en répondant aux exigences de sécurité. 
En matière de sécurité, une gestion du trafic plus sûre implique des recherches sur des systèmes mécatroniques, ainsi que des systèmes de contrôle élaborés pour la surveillance et le suivi des défauts de la voie.

Les recherches sur les composants électriques et caténaires demeurent également une voie d'innovation si l'on veut des trains allant plus vite. Il s'agit par exemple de réduire l'encombrement par l'utilisation de composants d'électronique de puissance. Ou encore d'étudier des systèmes électriques réduisant la maintenance tout en augmentant la performance, venant remplacer des systèmes pneumatiques existants pour la fermeture des portes, le freinage...

Il faut aussi considérer que depuis l'avènement du train, les vitesses et charges par essieu n'ont cessé d'augmenter, sans pour autant que le système constitué du train et de son environnement ne fassent l'objet de beaucoup d'investigations. Les solutions d'origine n'ayant presque pas changé, alors que de nombreux accidents récents montrent que ce changement s'impose. Le développement de modèles numériques pour mieux appréhender le comportement du système global constitué de la voie et du train est en conséquence un axe de progrès dans un secteur dans lequel ce type d'approche n'a pas été suffisamment mis en ouvre.

De même, la nécessité d'optimiser les interconnexions entre les différents modes de transport implique le développement de modèles logistiques prenant en compte la plurimodalité et permettant de simuler les flux de passagers ou de fret, dans la double perspective de réduire les délais et d'augmenter le trafic.

L'IRT Railenium a ainsi identifié un certain nombre de thématiques de recherche qui couvrent l'ensemble des composants et systèmes de l'infrastructure, les interfaces avec le matériel roulant ainsi que les activités s'y rapportant :

- les différents types de voies et leurs composants ;

- la maîtrise et la modélisation des contacts roue/rail ;

- l'élaboration de réseaux de capteurs intelligents pour l'état de la voie et la surveillance des points critiques de l'infrastructure ;

- le recours aux matériaux issus de ressources renouvelables ;

- les leviers pour augmenter la capacité d'une ligne ferroviaire ;

- l'optimisation des opérations de maintenance et de chantier ;

- la durabilité de la voie et de sa plate-forme ;

- les systèmes de localisation des trains ;

- la mise au point de réseaux de communication à très haut débit utilisant la technologie laser ;

- les systèmes de signalisation ferroviaire et leur interopérabilité.

\subsection{L'industrie navale et l'ingénierie maritime}

\subsubsection{Importance macroéconomique du secteur}

La mer recouvre $72 \%$ de la surface de la "planète Bleue », la Terre. Origine de la vie, elle influe de multiples façons sur notre existence. Climats et biodiversité sont, en particulier, fortement dépendants de la dynamique des océans. 
Mais la mer représente également un enjeu économique majeur pour l'avenir du monde.

Les échanges commerciaux de la planète se font à plus de $80 \%$ en volume par voie maritime grâce à 50000 navires de haute mer qui sillonnent en permanence les océans. Le transport maritime, qui a plus que doublé au cours des vingt dernières années, croît proportionnellement au PIB mondial. On prévoit $30 \%$ de croissance du transport de conteneurs d'ici à 2018. Pourtant, ce mode de transport ne génère que $3 \%$ des émissions de $\mathrm{CO}_{2}$ attribuées à l'ensemble des transports.

Plus de $95 \%$ des communications téléphoniques intercontinentales, dont notamment les liaisons de données et Internet, passent par les réseaux de câbles sousmarins. La pose, la surveillance et l'entretien de ces réseaux nécessitent une flotte câblière d'une centaine d'unités réparties principalement entre quatre nations : les États-Unis, le Japon, la France et le Royaume-Uni.

Première source de protéines animales pour l'alimentation humaine, les océans fournissent annuellement 163 millions de tonnes de poissons (chiffre 2009 de la $\mathrm{FAO}$ ), $55 \%$ en provenance de la pêche et $45 \%$ par aquaculture, une proportion en constante augmentation.

Les exploitations offshore produisent déjà plus de $30 \%$ des hydrocarbures, pétrole et gaz, chiffre en croissance puisque l'on estime que les champs sous-marins pourraient représenter jusqu'à $90 \%$ des réserves mondiales. Les réserves immergées de minerais, et notamment de métaux rares, sont estimées à plus de 200 fois les réserves terrestres connues.

Suivant certaines estimations, l'énergie de la mer, sous toutes ses formes - éolienne, hydrolienne, énergie des vagues, énergie thermique - pourrait produire annuellement 120000 TWh d'électricité, soit l'équivalent de la consommation totale de l'humanité.

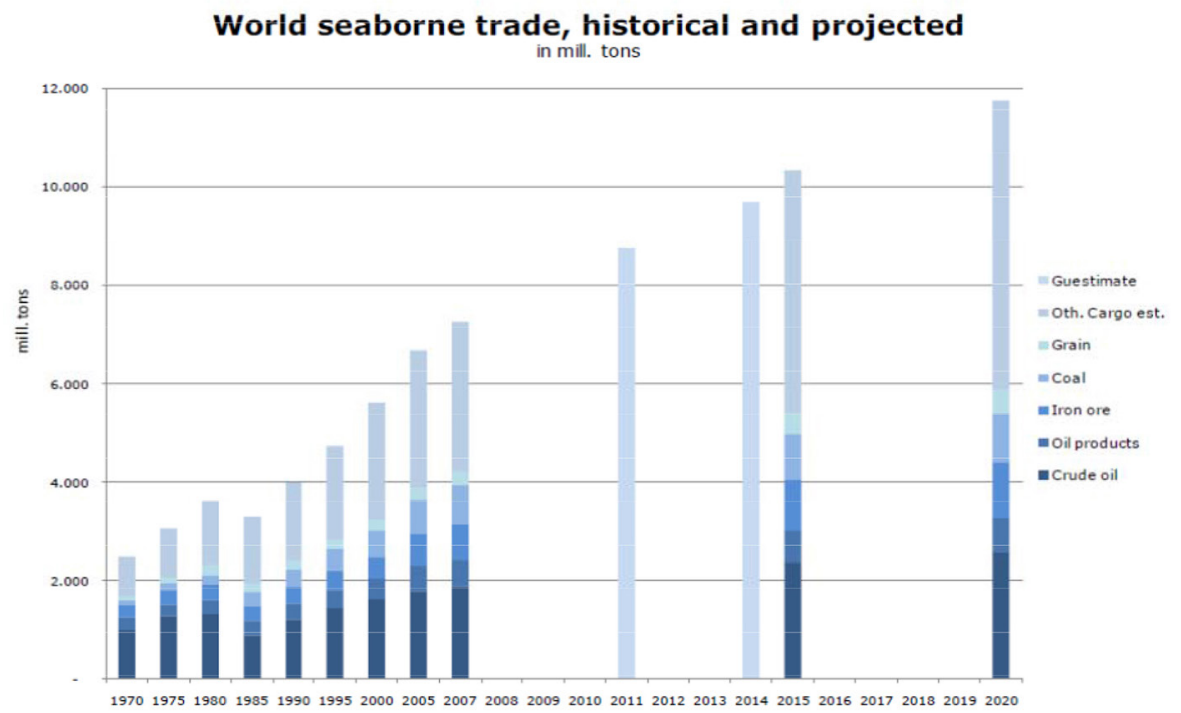

FIgURE 2.5. - Prévisions de croissance du trafic maritime. Source : CESA. 


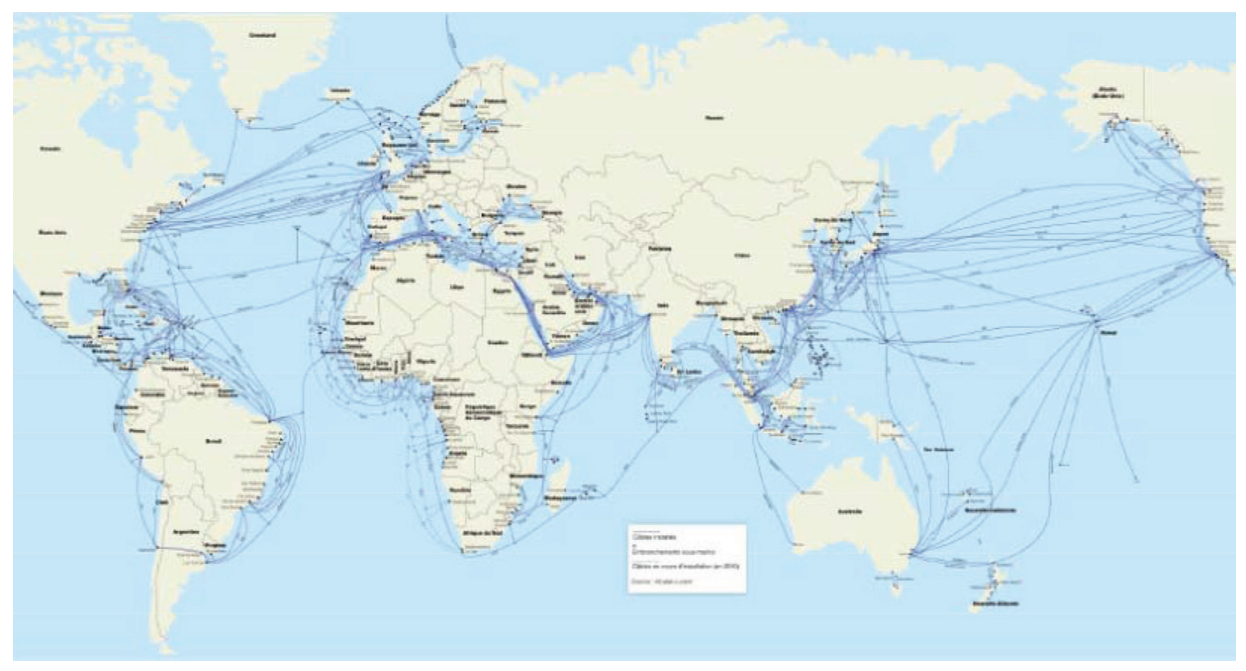

Figure 2.6. - Le réseau mondial de fibres optiques sous-marines (« Enjeux maritimes », Le dessous des cartes, Jean-Christophe Victor, Lépac, 2011).

L'économie maritime, sous ses différentes formes, pèse pour 1500 milliards d'euros dans le PIB mondial, la moitié au titre de l'extraction d'hydrocarbures et de minerais, l'autre moitié au titre du transport et des activités halieutiques. En Europe, les industries et services liés à la mer, au sens large mais hors hydrocarbures et pêche, génèrent un chiffre d'affaires de 500 milliards d'euros et occupent 5,4 millions de personnes (rapport Blue Growth de la Commission européenne).

Plus de 3,2 milliards d'êtres humains vivent aujourd'hui en zone littorale. À l'horizon 2025, $75 \%$ de la population mondiale se concentrera sur une bande côtière de moins de $80 \mathrm{~km}$ de large. Le besoin d'aménagement et d'exploitation d'installations offshore, pour la production d'énergie et de nourriture, pour la récupération et la transformation des matières premières issues des mers, pour la recherche biologique et pharmaceutique, pour les loisirs va donc croître de façon exponentielle.

Pour la France, qui possède le second plus grand domaine maritime au monde après les États-Unis avec 11 millions de $\mathrm{km}^{2}$ de zone économique exclusive, le secteur maritime représente 300000 emplois pour un chiffre d'affaires de 51 millions d'euros (Cluster maritime français).

L'importance croissante des activités maritimes pose de façon incontournable la question de leur régulation, de leur surveillance et de leur protection, dans un espace qui a été de tout temps considéré comme un espace de liberté échappant, pour l'essentiel, à toute souveraineté. Si la communauté internationale, à travers des organismes tels que l'OMI (Organisation Maritime Internationale) ou le Conseil de l'Arctique, établit progressivement les règles de vie commune, la défense des intérêts nationaux comme du droit international repose sur le déploiement de systèmes de surveillance et d'intervention armée. Le marché des navires militaires se monte à près de 40 milliards de dollars par an, dont environ une dizaine de milliards ouverts à la concurrence internationale. Ce marché de coopération militaire, incluant une forte proportion de transfert de technologie, 
est aujourd'hui dominé par les constructeurs européens (essentiellement français, allemands, espagnols et italiens).

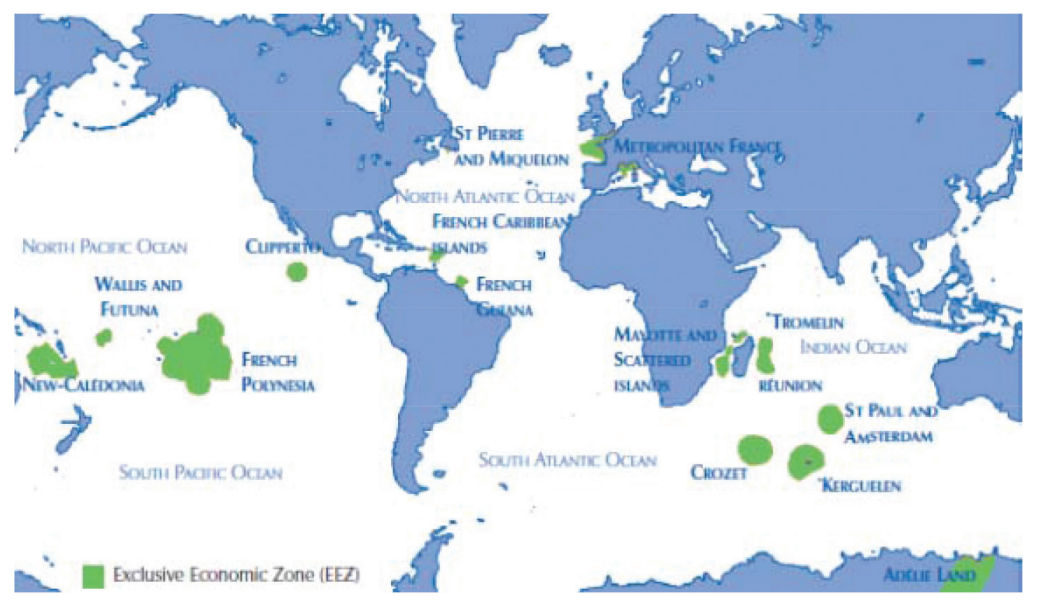

FigURE 2.7. - Zone économique exclusive (ZEE) française (Cluster maritime français).

\subsubsection{Tendances, enjeux et défis à l'horizon 2020}

La croissance de l'industrie navale, si l'on s'en tient aux activités d'ingénierie, de construction et de maintenance d'engins maritimes et d'installations océaniques, accompagnera l'augmentation des besoins en matière de transport - fret et passagers -, de loisirs - le marché de la croisière a doublé entre 1999 et 2009 -, de production d'énergie et de matières premières. Elle continuera inévitablement à subir les aléas cycliques de l'économie mondiale et du prix des hydrocarbures, ainsi que, sur certains segments de marché, de la concurrence agressive de pays émergents à faibles coûts de revient. Le marché naval est un marché de demande où les produits sont réalisés à l'unité, ou en très petit nombre, sur les spécifications particulières de chaque client. Les effets de série sont très faibles et ne justifient donc pas les mouvements de concentration qui ont pu être observés ces dernières années dans d'autres secteurs, notamment l'aéronautique. Ainsi, l'Association européenne des industriels du naval (SEA Europe) compte-t-elle plus de 300 chantiers adhérents dont près d'une trentaine de chantiers majeurs.

C'est donc dans le domaine des produits complexes à hautes exigences de performances que se sont aujourd'hui spécialisés les principaux constructeurs navals européens et particulièrement français : navires militaires, navires à passagers, ingénierie offshore, grande plaisance. Dans ces domaines, la compétitivité repose principalement sur l'effort de R\&D et la capacité d'innovation. Depuis quelques années, cette industrie mise également sur le développement de l'exploitation de l'énergie des mers (énergies marines renouvelables ou EMR). À plus long terme, l'exploitation et la transformation in situ, notamment à grande profondeur, des 
autres immenses ressources marines pousseront inéluctablement l'industrie à concevoir et développer de nouvelles installations. Dans un environnement naturel agressif et dangereux, la tendance sera de plus en plus forte de confier à des engins télécommandés (Remote Operated Vehicles ou ROV) ou autonomes (drones) les tâches répétitives (surveillance par exemple), les plus risquées (interventions à grande profondeur, actions militaires) ou pénibles (station prolongée sous l'eau).

Créé en 2011, à la suite du Grenelle de la mer, le Conseil d'Orientation de la Recherche et de l'Innovation des Constructions et Activités Navales (CORICAN) a identifié les grands défis techniques d'avenir. Ces défis résultent de l'évolution règlementaire internationale autant que de nouvelles exigences sociétales, notamment en matière de développement durable. Quatre grands programmes ont ainsi été définis : la maîtrise de l'énergie, le respect de l'environnement, l'amélioration de la sécurité et l'automatisation.

Comme pour tous les modes de transport, les économies de carburant, associées à la réduction des émissions polluantes imposée par les règles OMI et les directives européennes, constituent un enjeu majeur. Ainsi, le plafond d'émissions de soufre sera réduit de $90 \%$ à partir du $1^{\mathrm{er}}$ janvier 2015 en zones dites « d'émission contrôlée ", et de moitié partout ailleurs dans les eaux européennes à partir de 2020. Les plafonds d'émission des oxydes d'azote sont également réduits. L'OMI recommande par ailleurs une réduction de $25 \%$ des émissions de $\mathrm{CO}_{2}$.

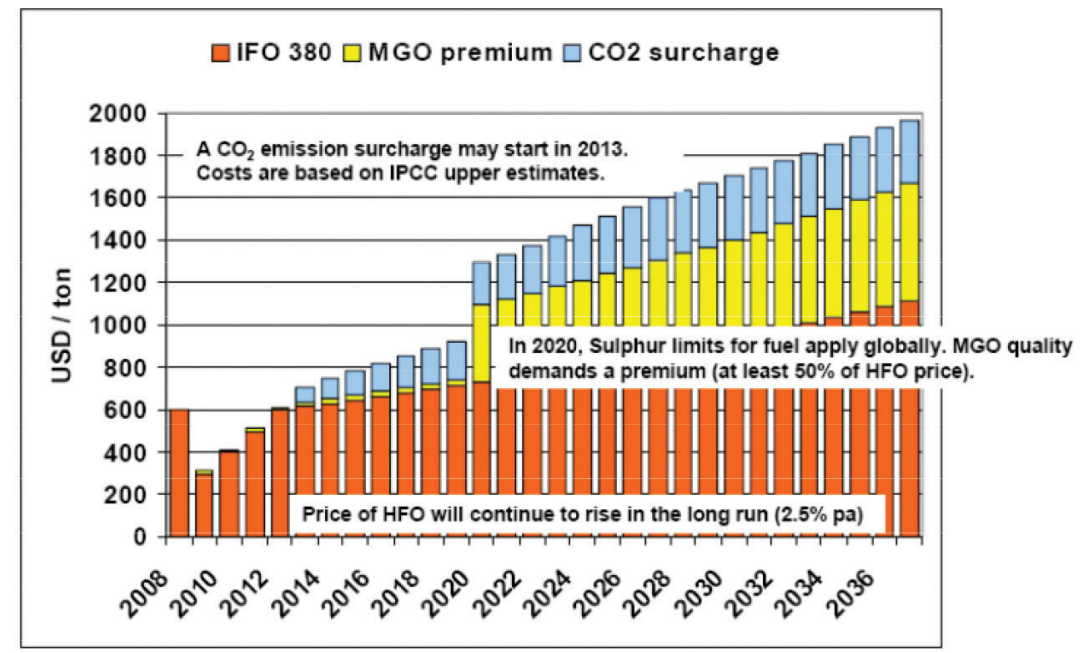

Source: GL research. The analysis excludes inflation effects.

Figure 2.8. - Projection du coût du combustible en fonction des nouvelles règles environnementales. La moitié de l'augmentation du coût dans les vingt prochaines années est liée à la règlementation environnementale. Source : GL research. The analysis excludes inflation effects.

L'impact environnemental de l'activité maritime ne se limite pas aux émissions gazeuses. Sont également concernés les revêtements et peintures, essentiels pour protéger de la corrosion les structures métalliques ou réduire le frottement sur 
les carènes, et qui sont soumis à la règlementation européenne REACH sur les produits chimiques. Quant à l'impact acoustique ou électromagnétique, étudié depuis plusieurs décennies pour les besoins militaires en raison de la formation de « signatures » indiscrètes, il est de plus en plus soumis à des limitations règlementaires pour réduire les perturbations causées à la biosphère marine.

L'océan est un milieu hostile et le travail en mer reste exposé aux dangers de cet environnement capricieux, isolé des moyens de secours rapides que l'on trouve généralement à terre (près de 2600 pertes de vies humaines en mer en 2013). Suivant leur taille - les pêcheurs payent toujours un lourd tribut à leur activité - ou leurs caractéristiques - tous les ans, des naufrages de ferries, souvent surchargés, font des centaines de victimes -, les navires sont confrontés à différents types de risques : risques naturels ou "fortunes de mer ", collision ou échouage, incendie, avarie résultant de l'état du navire ou de sa cargaison. En raison de l'intensité du trafic maritime en Manche/Mer du Nord, la France est un des pays les plus exposés aux catastrophes maritimes pour l'environnement.

La règlementation internationale, telle la convention SOLAS (Safety of Life at $S e a$ ), le renforcement de la surveillance du trafic maritime, notamment depuis la mise en service de l'AIS (Automatic Identification System), les normes de formation des gens de mer MLC (Maritime Labour Convention), l'amélioration des moyens de sauvetage en mer concourent à accroître la sécurité de l'activité maritime. Mais des progrès sont également attendus dans la conception des structures, en particulier en matière de stabilité et de tenue à la mer en conditions extrêmes ou exceptionnelles, de tenue à la fatigue, aux chocs, aux impacts, d'optimisation adaptative des conditions de navigation (cap, vitesse) aux conditions de mer, ce qui, dans tous les cas, exige une meilleure connaissance et, surtout, une capacité de prévision en temps réel de la houle de rencontre.

\subsubsection{Attentes vis-à-vis des partenaires-fournisseurs équipementiers-ensembliers}

Pour la première fois, sans doute, de façon aussi structurée, la démarche prospective engagée par le CORICAN est un travail de filière qui associe l'ensemble des acteurs. Les objectifs cités plus haut sont donc portés par les industriels intégrateurs, DCNS et STX France, aussi bien que par les équipementiers. Pour atteindre ces objectifs, de nombreuses démarches collaboratives se construisent dans le cadre, notamment, des pôles de compétitivité - pôles Mer et EMC2 -, du Programme d'investissements d'avenir - programme " Navire du futur », institut de recherche technologique Jules-Verne -, des programmes de recherche européens - exemple du programme AQUO sur l'impact acoustique du trafic maritime sur la faune marine.

L'amélioration de l'efficacité énergétique et la réduction des émissions polluantes passent, en grande partie, par l'amélioration des systèmes de production d'énergie et de propulsion. En particulier, le remplacement du gazole par le gaz naturel est un enjeu fort pour les motoristes comme pour les concepteurs de navires qui doivent imaginer les moyens sûrs de stockage à bord. Le recours, fût-il de façon auxiliaire, à d'autres sources d'énergie comme les piles à combustibles, l'énergie solaire ou éolienne, les batteries Li-Ion, est également visé. 
L'automatisation de l'ensemble des fonctions à bord - navigation, conduite, commande des différentes installations, maintenance - est un enjeu de sécurité et de réduction des coûts d'exploitation. Elle passe par l'introduction massive de réseaux de capteurs et de technologies de l'information basées sur des algorithmes de traitement et d'aide à la décision de plus en plus élaborés. Le projet de « passerelle du futur » associe un nombre important d'équipementiers et de fournisseurs de la filière.

Le développement de technologies pour les engins autonomes telles que les communications sous-marines à haut débit, le stockage d'énergie, les algorithmes d'analyse en temps réel permettant de conférer aux drones une autonomie décisionnelle, les moyens mécaniques de lancement et récupération de ces engins, doit être poursuivi.

Enfin, les outils et procédés de production des structures, EMR, plates-formes ou navires doivent évoluer pour s'adapter aux objectifs de compétitivité : réduction des temps de fabrication et des taux de reprises, intégrations de nouveaux matériaux, notamment composites, réalisation de moyennes séries. L'amélioration de la sécurité en ateliers, sur les chantiers ou en mer doit aussi être prise en compte. Cela passera largement par la robotisation et l'informatisation des procédés dont l'étude est en cours dans le cadre du projet de " chantier du futur », l'un des volets du plan « usine du futur» de la nouvelle France industrielle.

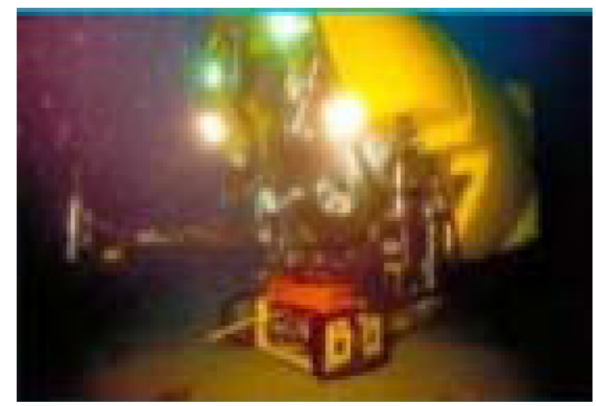

FIGURE 2.9. - Engin sous marin autonome.

\subsubsection{Domaines clés de recherche et d'innovation}

Plus ancienne industrie au monde, la construction navale est aussi la première activité humaine à être passée, dans le courant du XVIII ${ }^{\mathrm{e}}$ siècle, du stade d'artisanat empirique et héréditaire à celui d'industrie fondée sur des concepts et des outils scientifiques. Ces bases scientifiques, la mécanique des fluides et la résistance des matériaux principalement, connurent alors une révolution « copernicienne » grâce aux travaux théoriques des meilleurs esprits de l'époque. Les essais de maquettes, réalisés en 1775 par d'Alembert, Condorcet et l'abbé Bossut, préfigurent le développement de la similitude expérimentale, formalisée un siècle plus tard par Frédéric Reech et William Froude.

Longtemps, la conception des navires s'est fondée sur les essais de maquettes et des règles de dimensionnement largement issues de l'expérience. La nécessaire 
réduction de la durée des phases de conception, l'optimisation des performances et la réduction des coûts qui passe par la réduction de marges de conception superflues contraignent aujourd'hui à développer de nouveaux outils de conception.

Les premiers progrès attendus concernent le domaine de l'hydrodynamique : résistance à l'avancement, prévision de houle et tenue à la mer, manœuvrabilité. Il est important, dans ce domaine, de développer les moyens de calcul numérique et d'en valider l'application de façon, à terme, à remplacer les expérimentations, ce que l'on appelle le "bassin numérique ». De plus, la simulation numérique, par l'efficacité des méthodes d'optimisation qu'elle autorise, permettra de tester et valider de nouveaux concepts de formes de carènes et d'appendices. Les codes de CFD actuels doivent être améliorés pour décrire encore plus finement les écoulements à dynamique rapide ou fortement turbulents. Ils doivent être étendus au couplage fluide/structure, notamment pour les simulations acoustiques ou l'optimisation des propulseurs. La recherche de revêtements réducteurs de frottements doit se poursuivre, ainsi que l'étude de propulseurs à hautes performances (rendement, bruit).

La simulation de la dynamique des structures, sollicitées par des chargements fortement variables dans l'espace et le temps, doit aussi progresser afin de mieux appréhender les comportements dangereux en instantané ou en fatigue. De plus en plus, ces simulations doivent être capables de représenter des structures complexes, multi-matériaux, métalliques et composites.

Dans le domaine des matériaux, la caractérisation en environnement de matériaux métalliques innovants (les applications du titane sont d'un intérêt particulier) doit se poursuivre dans le but d'alléger les structures, d'améliorer la tenue à la corrosion et à la fatigue, d'améliorer la productivité des procédés de formage (fonderie, métallurgie, formage à chaud, méthodes additives, technologie des poudres), d'usinage, d'assemblage (soudage, collage) et de contrôle (contrôle non destructif). La maîtrise de la qualité métallurgique des pièces finies passe également par un effort de simulation numérique des différents procédés mis en œuvre depuis la matière initiale.

Les modes d'initialisation de la corrosion, la simulation des endommagements et les dispositifs de protection - par revêtements ou par courant imposé - doivent continuer à être étudiés, notamment dans la perspective de limiter les coûts de maintenance de structures offshore fixes.

L'emploi de matériaux composites se développe dans la construction de superstructures et de structures internes (cloisons, planchers) dans les navires, et pour les pièces mobiles (pales) de propulseurs ou d'éoliennes et hydroliennes. La tenue de ces matériaux aux agressions - chocs, incendie - et au vieillissement doit être améliorée. Des systèmes de surveillance en continu de l'état de telles structures doivent également être envisagés, associés à des modèles de prédiction de durée de vie.

Les matériaux multifonctions, ou métamatériaux insèrent différents composants ou additifs, incluant des nanomatériaux, dans une matrice organique structurale pour remplir d'autres fonctions : émission/réception de signaux électromagnétiques, masquage acoustique, protection contre les incendies ou les rayonnements... Leur définition, leur caractérisation, les conditions et procédés de mise en œuvre restent largement à étudier. 
Enfin, l'un des défis les plus complexes réside dans la transition rapide et sûre des innovations technologiques dans la conception et la construction des navires. Cela suppose un effort continu dans l'amélioration des outils de conception, basée sur la simulation numérique (le « navire virtuel », l'échange étendu de données et l'optimisation multiphysique et multiobjectifs).

Enfin, dans le domaine de la production, quantité de travaux sont initiés, mais sont loin d'avoir atteint le stade d'industrialisation, dans le domaine de la robotisation des procédés - la mécatronique - en environnement contraint, sur l'association Homme/robot - la cobotique -, dans l'utilisation industrielle de la réalité virtuelle avec simulation d'efforts - procédés haptiques - et de la réalité augmentée.

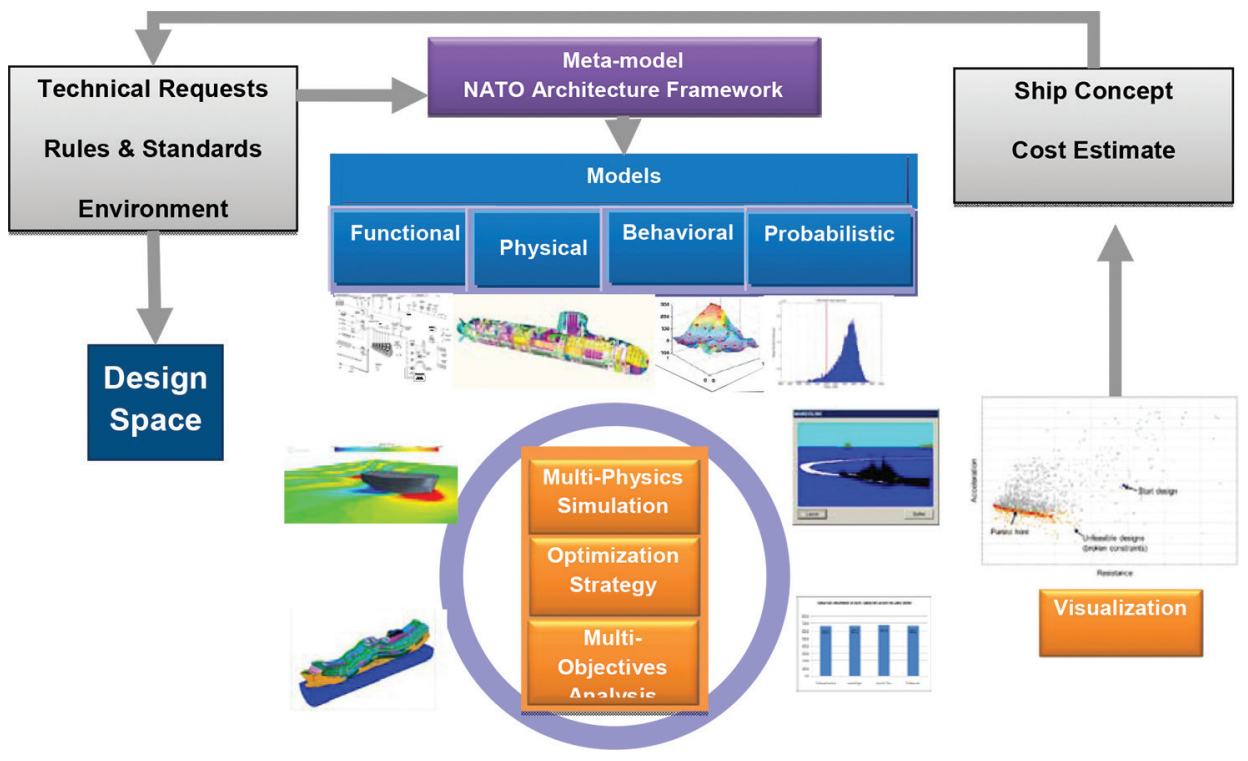

Figure 2.10. - Démarche de R\&D en ingéniérie maritime.

\subsection{L'industrie agroalimentaire}

\subsubsection{Importance macroéconomique du secteur}

Le secteur des Industries Agro-Alimentaires (IAA) regroupe :

- en amont : les activités de transformation des matières premières agricoles en produits alimentaires intermédiaires ou finis, comestibles par les humains ou les animaux ;

- en aval: les activités de transformation ou d'assemblage de produits intermédiaires. 
Le secteur, composé de huit grandes filières, est organisé en fonction des produits fabriqués ou transformés :

- industrie de la viande: abattage du bétail, de la volaille, charcuterie, conserverie ;

- industrie laitière : fabrication du lait, du beurre, des yaourts, des fromages, du lait en poudre ou concentré, des glaces, fractionnement du lait pour l'industrie alimentaire (caséine, lactose, protéines ultrafiltrées), etc. ;

- fabrication de produits alimentaires élaborés : fruits, légumes, poissons, plats cuisinés et confitures ;

- fabrication de produits à base de céréales : farine, pain et pâtisseries industriels, biscuits, biscottes, semoules et pâtes alimentaires, céréales pour le petit déjeuner, malt, amidon, fécules et produits dérivés, aliments pour animaux, etc. ;

- fabrication d'huiles, de corps gras et de margarines ;

- industrie sucrière ;

- fabrication de produits alimentaires divers : chocolat, confiseries, café et thé conditionnés, épices, vinaigres, sauces préparées, aliments pour bébés, produits de régime, desserts, bouillons, levures, etc. ;

- fabrication de boissons et alcools : vins, eaux de vie, distillation d'alcool, apéritifs, champagne, bière, jus de fruits et de légumes, eaux minérales, etc.

Le secteur des IAA est le premier secteur industriel mondial. Il représente près de $4 \%$ du PIB de la planète et emploie au total 22 millions de personnes. Au niveau de l'UE, le secteur agroalimentaire est aussi le premier secteur manufacturier, avec un CA de 965 milliards d'euros (13\% du CA de l'industrie manufacturière européenne) et un effectif de 4,4 millions de salariés.

En France, le secteur des IAA est également le premier secteur industriel. Il contribue à environ $19 \%$ du chiffre d'affaires et des effectifs salariés de l'ensemble du secteur manufacturier français, avec un CA de 159 milliards d'euros et 576000 salariés (chiffres de 2010). À noter que les filières « viande » et « laitière » représentent $38 \%$ du CA et $41 \%$ des effectifs salariés. En outre, le secteur des IAA contribue à $16,5 \%$ de la valeur ajoutée de l'ensemble du secteur manufacturier français, soit environ 35 milliards d'euros. À noter que les filières «boissons et alcools » et «produits alimentaires divers » représentent plus de $40 \%$ de la valeur ajoutée.

En 2011, les exportations agricoles et agroalimentaires françaises ont totalisé 57 milliards d'euros (dont 41 milliards d'euros pour les produits des IAA), soit $13 \%$ des exportations françaises, induisant un solde excédentaire de la balance commerciale de 11,7 milliards d'euros, en deuxième position derrière le secteur de l'aéronautique. À noter que la filière «boissons et alcools » représente environ $30 \%$ des exportations. La part de marché des exportations agroalimentaires françaises au niveau mondial est de $5,4 \%$, ce qui place la France au quatrième rang derrière les États-Unis, les Pays-Bas et l'Allemagne. À noter que la France est en recul relatif par rapport à ses concurrents européens et aux BRICs qui progressent plus vite. Ainsi, sur les dix dernières années, la France est passée du premier au quatrième rang mondial. 
Ces chiffres montrent à l'évidence que les IAA occupent au sein des industries manufacturières une place essentielle dans les économies européenne et française. En France, les États généraux de l'industrie (conclus en mars 2010) ont identifié le secteur agroalimentaire comme l'un des dix secteurs industriels stratégiques au niveau national. Le secteur contribue de manière très positive au commerce extérieur de la France et se caractérise par une certaine robustesse au sein du secteur industriel, avec une relative stabilité des effectifs salariés. Toutefois, les IAA françaises se développent et se structurent insuffisamment, ce qui entraîne une perte de compétitivité par rapport à leurs homologues dans le monde. Cette caractéristique structurelle explique en grande partie le recul relatif de la France au plan mondial.

\subsubsection{Enjeux et défis des IAA à horizon 2020}

Dans un marché mondial marqué par l'accélération des changements économiques (globalisation de l'économie, libéralisation du marché) et des développements technologiques, les IAA doivent faire face à une compétition accrue des pays émergents (BRICs notamment), aux contraintes réglementaires qui évoluent vite (qualité, hygiène, sécurité) et aux attentes plurielles des consommateurs en matière d'alimentation (qualité, diversité, santé, bien-être, accessibilité). Tous ces facteurs impactent fortement et directement les activités, l'organisation et l'emploi dans le secteur des IAA. Pour les IAA européennes et françaises, ils déterminent les enjeux et défis des IAA à horizon 2020 et au-delà.

Dans un contexte de plus en plus concurrentiel, les IAA européennes et françaises sont encore leaders dans de nombreuses filières. Mais, ce leadership pourrait se fragiliser à moyen/long terme si des mesures volontaristes n'étaient pas prises dès maintenant pour améliorer la capacité d'innovation multidisciplinaire des IAA. Ce constat se trouve renforcé par les éléments suivants :

- les IAA revendiquent une vraie valeur ajoutée dans la prévention des maladies liées au style de vie et de consommation. Mais, cette valeur ajoutée n'est réellement effective dans l'alimentation que si de nouveaux concepts produits-procédés sont développés et exploités, ce qui requiert des investissements R\&D conséquents et ciblés ;

- la concurrence des pays développés et émergents (États-Unis, Japon, BRICs) qui considèrent l'innovation dans les IAA comme un axe stratégique pour leur développement économique et social. À ce titre, l'innovation dans les IAA est pleinement intégrée dans la politique industrielle de ces pays ;

- la concurrence des pays à bas coût (BRICs) s'accroît. Même si la maîtrise des coûts demeure un objectif permanent et incontournable pour les IAA européennes et françaises, leur avenir à moyen/long terme réside surtout dans le développement et la production de produits à haute valeur ajoutée, en s'appuyant sur un niveau d'innovation soutenu et une avance technologique différenciante ;

- les efforts de R\&D des IAA européennes et françaises ont été jusqu'à présent fragmentés et relativement faibles. En général, les PME investissent peu en R\&D. Et les grands groupes tendent à concentrer leurs efforts sur 
le marketing. À noter que l'investissement R\&D en Europe et en France est de l'ordre de $0,4 \%$ du CA et de $1,5 \%$ de la valeur ajoutée, respectivement ; ce qui est très faible comparé aux autres secteurs manufacturiers. Les IAA privilégient l'innovation incrémentale, qui repose sur la modification et l'amélioration de produits et de procédés. L'innovation de rupture qui consiste en la mise sur le marché d'un produit totalement nouveau est plus rare. Les IAA déposent peu de brevets (en moyenne 200 à 250 brevets par an en France). En Europe comme en France, il est nécessaire de définir et mettre en place des stratégies R\&D long terme pour favoriser les ruptures en matière de produits et de procédés ; et ce, pour sécuriser et consolider le leadership européen et français sur le long terme.

En Europe comme en France, compte tenu des enjeux et des défis mentionnés ci-dessus, les pouvoirs publics et le secteur des IAA s'accordent sur la nécessité de cibler des axes prioritaires pour préparer l'excellence technologique (les ruptures technologiques notamment), et ainsi sécuriser une industrie performante et compétitive s'appuyant sur une économie en croissance, une richesse technologique partagée, une production alimentaire durable et la confiance des consommateurs.

Ces axes prioritaires sont les suivants :

- accroître l'investissement de R\&D, ainsi que la vitesse et la qualité d'innovation ;

- activer la collaboration entre les différents intervenants des filières industrielles ;

- optimiser l'acquisition de connaissances et activer le transfert de connaissances vers les PME;

- adapter/incorporer des concepts modernes de production, notamment en se ressourçant auprès d'autres secteurs manufacturiers performants.

La stratégie de développement à long terme du secteur des IAA européennes et françaises est donc clairement basée sur la recherche et l'innovation, qui ont pour ambition de préparer les ruptures technologiques dans ce secteur et d'associer les différents acteurs (IAA, partenaires-fournisseurs industriels, instituts de recherche).

\subsection{3. Équipements, procédés, lignes de production dans les IAA : moteurs de l'innovation}

Le leadership des IAA européennes et françaises constitue l'un des atouts majeurs pour les équipementiers-ensembliers européens et français. En effet, le secteur des IAA représente pour les équipementiers-ensembliers à la fois un marché considérable (marché intérieur et marché à l'export) et un formidable potentiel de différenciation technique et technologique grâce à la capacité d'anticipation des filières industrielles leaders.

Compte tenu d'une part du contexte très concurrentiel du marché agroalimentaire mondial, et d'autre part des axes prioritaires visant à préparer l'excellence technologique comme facteur de différenciation, les IAA ne préserveront et consolideront durablement leur leadership dans le monde que si elles collaborent efficacement avec leurs partenaires équipementiers-ensembliers. Il s'agit là de promouvoir 
des alliances gagnant-gagnant sur le moyen/long terme entre IAA et équipementiers-ensembliers, ces derniers étant considérés non seulement comme des fournisseurs potentiels d'équipements, mais aussi comme des porteurs de valeur ajoutée technologique (grâce à leurs savoir et savoir-faire reconnus sur les équipements et procédés, voire sur les produits). À charge pour les équipementiers-ensembliers de mettre en place des stratégies d'alliance gagnant-gagnant avec les IAA et de s'organiser pour mettre leurs capacités de création, d'innovation et de réalisation au service du développement industriel agroalimentaire, au plan local comme mondial.

Afin d'éclairer les équipementiers-ensembliers en matière de $R \& D$ nécessaire pour accompagner efficacement le développement dans le secteur des IAA, il convient de décliner les orientations prioritaires de ce secteur en matière de R\&D à long terme (dans l'UE et en France).

\subsubsection{Le développement durable}

À l'image des autres secteurs industriels, le secteur des IAA s'engage vers des stratégies de développement durable qui associent la prise en compte des dimensions économiques, écologiques, sociales et sociétales. Les défis environnementaux auxquels les IAA doivent faire face consistent notamment à :

- maîtriser la consommation d'énergie et assurer une meilleure efficacité énergétique des systèmes de production ;

- valoriser au maximum la ressource agricole et réduire les déchets ;

- anticiper les effets du changement climatique et réduire les émissions de gaz à effet de serre (GES) ;

- réduire et optimiser la consommation d'eau dans les processus de fabrication et veiller à la qualité des rejets des eaux usées ;

- limiter tous les transferts de polluants dans les milieux eau/sol/air ;

- contribuer à la valorisation des déchets, accroître la performance environnementale des emballages en lien avec la politique de gestion des déchets.

Avec le développement et l'industrialisation d'équipements et de procédés intensifiés écoconçus, les équipementiers-ensembliers sont capables d'apporter des réponses très concrètes et différenciantes, en lien avec les enjeux stratégiques du développement durable dans les IAA. Dans le prolongement de cette orientation, les équipementiers-ensembliers peuvent aussi contribuer au développement de systèmes de production intelligents (intégration des technologies de l'information et de la communication, ou TIC, dans les processus de transformation et de production) afin de rendre ces systèmes plus productifs et plus flexibles; avec le double objectif de mettre ces avancées technologiques à la portée non seulement des grands groupes agroalimentaires, mais aussi des PME du secteur.

\subsubsection{Qualité et hygiène alimentaires}

L'alimentation est étroitement liée aux notions de plaisir, bien-être et santé. Associée aux allégations santé grâce à un contenu nutritionnel optimisé, l'attribut sensoriel lié à la saveur d'un aliment s'avère être très important pour le consommateur. En outre, les changements sociétaux et l'évolution des habitudes alimentaires 
qui en découle (travail des femmes, vieillissement de la population, intégration croissante de groupes ethniques, etc.) dynamise la créativité et le développement des «aliments service ». Enfin, la notion de diversité alimentaire connaît une importance accrue dans le développement de nouveaux produits.

Face aux nombreuses crises qui ont touché la filière agroalimentaire («vache folle », dioxine, etc.), l'Union Européenne a achevé l'harmonisation d'une réglementation, dite " paquet hygiène », pour assurer la sécurité sanitaire de l'alimentation humaine et animale. Il s'agit d'obtenir un niveau élevé de protection du consommateur tout en tenant compte des enjeux économiques (la libre circulation des produits) et culturels.

La R\&D orientée produits (qui intègre la nutrition, les sciences des aliments, le génie de la formulation, les sciences de la consommation, etc.), aussi puissante soitelle, ne peut pas à elle seule satisfaire complètement et durablement les exigences croissantes en matière de qualité et d'hygiène alimentaires. Une R\&D orientée équipements et procédés est alors nécessaire pour atteindre les objectifs de compétitivité durable des IAA européennes et françaises. Là, les équipementiers-ensembliers ont évidemment un rôle important et complémentaire à jouer pour concevoir et développer les nouvelles technologies de transformation des produits alimentaires (équipements, procédés et lignes) adaptées aux exigences de qualité et d'hygiène alimentaire, qui évoluent, capables de servir à la fois le marché local (national, régional) et les marchés à l'international. À titre d'exemples, on peut citer les technologies douces de préservation et de séparation, les biotechnologies, les micro- et nanotechnologies appliquées à la texturation alimentaire, les traitements thermomécaniques de milieux complexes concentrés, etc.

\subsubsection{Axes de recherche et d'innovation pour les équipementiers-ensembliers}

Les paragraphes précédents mettent clairement en évidence un contexte ambitieux visant à renforcer et à consolider la compétitivité et le leadership du secteur IAA en Europe comme en France. Basée sur un effort sur le long terme de recherche et d'innovation, cette ambition vise non seulement l'innovation incrémentale, mais aussi et surtout l'innovation de rupture, afin de s'approprier les ruptures technologiques nécessaires du secteur des IAA, en lien avec les orientations prioritaires présentées ci-dessus.

Tout d'abord, il convient de rappeler que le développement technologique requiert des compétences pluridisciplinaires qui relèvent non seulement du génie des équipements, mais aussi du génie des procédés (sciences de l'ingénieur qui décrivent les fonctions procédés des équipements). Les deux domaines sont très complémentaires et, plus que jamais, il importe d'associer les expertises techniques et scientifiques des deux domaines pour contribuer à relever à un meilleur niveau les défis technologiques des IAA, et conférer une valeur ajoutée fonctionnelle aux matériels et équipements de procédés. Aussi est-il capital pour les équipementiers-ensembliers du secteur des IAA de s'appuyer, dans le développement de leurs équipements et matériels, sur des compétences avancées en génie des équipements et en génie des procédés. Sont listés ci-après quelques axes essentiels de recherche et d'innovation 
qui devraient permettre aux industriels de la mécanique de répondre efficacement et concrètement aux besoins de développement technologique des IAA.

\subsubsection{Génie des équipements}

- Écoconception et conception hygiénique des matériels et équipements de procédés ;

- robotique et mécatronique ;

- rendements (énergie, matière) et flexibilité (agilité/rapidité pour les changements de lots, de recettes et de processus de fabrication) des matériels et équipements de procédés ;

- sécurité des matériels et équipements de procédés.

\subsubsection{Intensification des procédés}

C'est un concept récent portant sur l'amélioration de procédés existants ou le développement de procédés nouveaux, visant à réduire l'impact environnemental et l'intensité capitalistique des procédés et lignes de production. Le but ultime est d'évoluer vers des technologies durables, en cohérence avec la définition du développement durable proposée par la WCED, World Commission on Environment and Development (rapport Brundtland, 1987) : "Le développement qui satisfait les besoins de la génération présente sans compromettre la capacité des générations futures à satisfaire leurs propres besoins. »

Les bases de l'intensification des procédés ont été établies ces dernières années par la communauté scientifique de génie des procédés et l'industrie des procédés (l'industrie chimique notamment). Ces bases reposent sur trois grands principes :

- promouvoir les procédés continus ;

- développer des équipements de procédés multifonctionnels ;

- accélérer les phénomènes de transfert (transferts de masse, chaleur et quantité de mouvement) et les dynamiques de conversion de la matière (conversions physico-chimiques).

Appliquée aux procédés et lignes de production du secteur des IAA, l'intensification des procédés devrait permettre de réduire l'impact environnemental (réduction des consommables, énergie et eau ; minimisation des déchets), d'accroître la productivité (augmentation des rendements matière), d'améliorer la sobriété et la sécurité des unités, et de réduire les coûts (coûts opératoires et coûts d'investissement). L'industrie chimique est déjà bien avancée dans l'exploitation de l'intensification des procédés. Un transfert de connaissances et de savoir-faire méthodologique dans ce domaine, au profit des IAA, doit être vivement encouragé.

\subsubsection{TIC et systèmes de production intelligents}

- Intégration des TIC dans la gestion opérationnelle de la production (opérations unitaires, lignes de production, chaînes alimentaire et logistique, 
traçabilité, suivi en temps réel de la qualité des produits, de l'empreinte carbone, de l'impact environnemental) ;

- TIC pour la modélisation/simulation des systèmes de production (notion d'usine virtuelle) pour développer des outils d'aide à la décision et à la formation (e-learning).

\subsubsection{Thermorhéologie des milieux complexes concentrés}

Les milieux rencontrés dans les IAA sont souvent très complexes et hétérogènes (systèmes diphasiques liquide/liquide, gaz/liquide, solide/liquide), eu égard aux formulations multicomposants mises en œuvre. Avec le développement d'équipements et de procédés intensifiants, on prévoit que ces milieux seront significativement plus concentrés (procédés de séchage, procédés de cuisson, etc.). Aussi convient-il de promouvoir les recherches visant à explorer et décrire les comportements thermorhéologiques de ces milieux. À cet effet, l'adaptation de méthodes existantes, voire le développement de nouvelles méthodes de mesure, doivent être envisagés.

\subsubsection{Métrologie et contrôle des procédés}

- capteurs en ligne (thermorhéologie) ;

- biocapteurs ;

- outils de diagnostic et imagerie (imagerie thermique, ultrasons, tomographie, systèmes acoustiques non invasifs) ;

- automation avancée des équipements, des procédés et lignes de production.

\subsubsection{Nouveaux matériaux fonctionnels}

- écoemballages ;

- emballages intelligents ;

- surfaces antimicrobiennes.

\subsubsection{Bibliographie}

Parmi les sources d'information consultées pour étayer cette analyse relative à l'industrie agroalimentaire, citons les deux documents :

- «Panorama des industries agroalimentaires ", Ministère de l'Agriculture, de l'agroalimentaire et de la forêt, Direction générale des politiques agricole, agroalimentaire et des territoires, Bureau des industries agroalimentaires, édition 2012, Paris, France, 64 pages.

- F. Gorga, M. Notarfonso, FoodManufuture, European Commission, KBBE2011-5-289327, deliverable $\mathrm{n}^{\circ} \mathrm{D} 2.1,2012,18$ pages. 


\subsection{L'industrie des technologies de l'environnement}

\subsubsection{Importance macroéconomique du secteur}

Aborder le thème de l'environnement selon l'axe des industries ou des technologies environnementales ne dispense pas de considérer leur impact, leur mise en œuvre et les conditions de réduction de ces impacts comme des sujets aujourd'hui très sensibles et sociétaux.

Un ministère ad hoc et un commissariat général en charge du développement durable ; les DREAL, des directives européennes, l'Echa sont bien là pour informer, mesurer et s'assurer de la bonne mise en place de dispositifs sévères, mais utiles et souvent nécessaires.

La diversité des milieux concernés - air, eau, sol et sous-sol, biodiversité, paysages, etc. -, les pressions et impacts sur l'environnement divers par leurs origines agriculture, sylviculture, pêche, construction, industrie, ménages, tourisme, transports, etc. - ont façonné les mentalités et entraîné progressivement une prise de conscience qui se traduit en nouvelles exigences.

La perception des risques devient générale, jusqu'à l'abus du principe de précaution, et modifie les pratiques environnementales, tant les comportements individuels que ceux des ménages ou des acteurs économiques.

C'est dans ce contexte que s'est mise en place toute une économie de l'environnement ou écoactivités pour rejoindre et reprendre les terminologies du MEDDE, ses définitions et présentations :

«La protection de l'environnement vise à prévenir, diminuer les émissions de polluants et les autres dégradations causées à l'environnement [...]. La gestion des ressources naturelles vise à diminuer les prélèvements sur les ressources naturelles : développement des énergies renouvelables, maîtrise de l'énergie, récupération, gestion durable de l'eau [...].»

Le marché international est évalué à 1000 milliards d'euros et le marché global français à 50 milliards d'euros. La France occupe le cinquième rang mondial à l'exportation avec $80 \%$ d'export vers les pays de l'OCDE et 8 milliards d'euros de productions.

À partir de 2015, Eurostat collectera annuellement les données de l'année N - 2 sur les écoactivités et la liste des produits comptabilisés dans la production; le commerce extérieur et la valeur ajoutée seront enfin pris en compte.

Les écoactivités relèvent d'ores et déjà pour l'essentiel d'entreprises marchandes, et les productions et les emplois liés peuvent être extraits du dispositif ÉSANE de l'INSEE.

Nous proposons de simplement rappeler les chiffres en termes d' emplois par domaine » générés sans les développer, en renvoyant le lecteur intéressé sur les sites dédiés du MEDDE (observations et statistiques), mais de flécher les différents domaines retenus tels qu'ils peuvent ressortir des planches (source «Production 2009-2011» du SOeS, données 2013). 


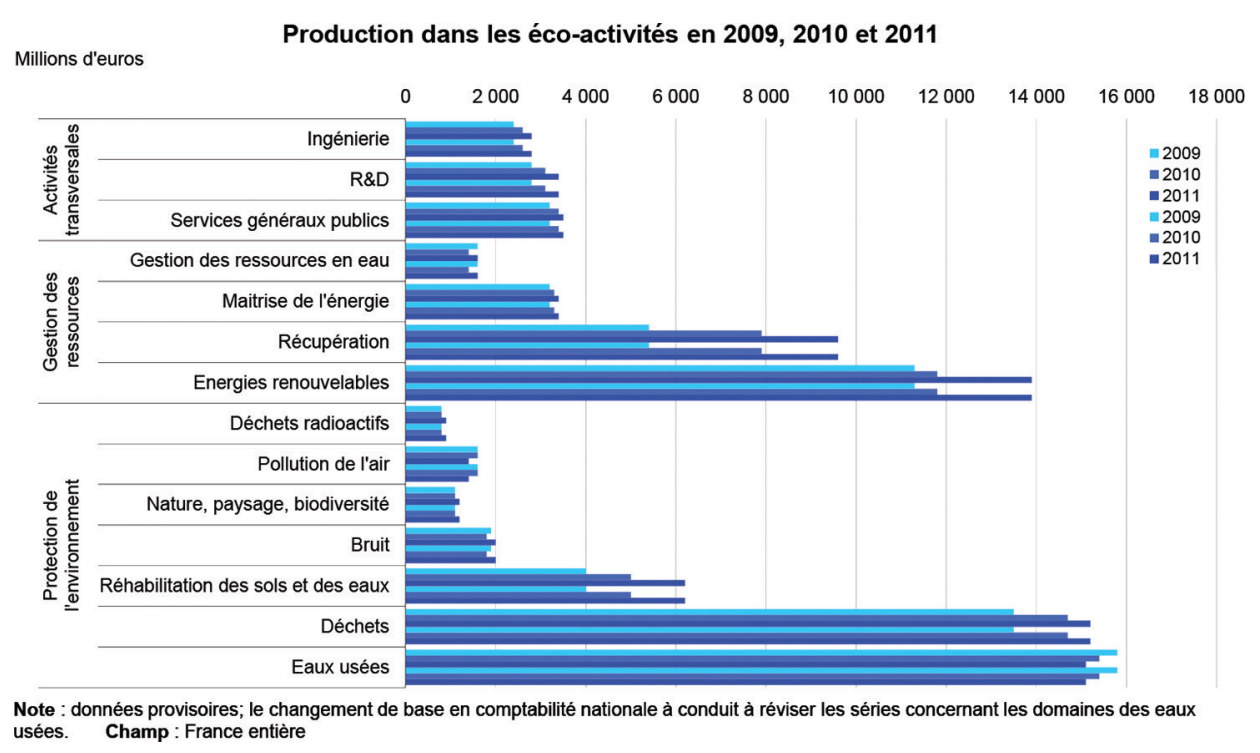

Figure 2.11. - Répartition des éco-activités. Source : SOeS, 2013.

\subsubsection{Tendances, enjeux et défis du secteur à l'horizon 2020}

La raréfaction générale des ressources traditionnelles, et des eaux de qualité en particulier, les prélèvements dans le milieu naturel qui restent excessifs, le prix d'accès aux énergies fossiles toujours en augmentation sont directement la conséquence des bouleversements climatiques et de l'augmentation des populations, qui restent des tendances lourdes sans inflexion.

Dans ces conditions, des risques sanitaires persistants, voire des risques sociaux, adviendront dans la mesure où l'accès aux ressources pour tous et la compétition dans les usages ou entre territoires n'est plus assurée.

Cette dynamique de fond aura un poids économique de plus en plus considérable et les marchés liés à l'environnement, qui représentent déjà un pourcentage conséquent du PIB mondial, voisin de $2 \%$, ne peuvent que croître rapidement.

Si le Livre Blanc de la Recherche en Mécanique n'est pas le cadre adapté pour développer ces hypothèses, il résulte de toutes ces contraintes de véritables opportunités de développement pour le secteur des services à l'industrie mécanique et à certaines spécialités des constructions mécaniques, car nous ne sommes qu'au début d'un processus de mutation de nos économies.

Le nombre d'écoentreprises est évalué entre 10000 et 12000 , de toutes tailles, mais avec des caractéristiques de taille bien précises selon les secteurs ou spécialités.

À côté des grands opérateurs, majors ou leaders mondiaux historiques, qui réalisent environ $40 \%$ du chiffre d'affaires global des écoactivités (source ADEME), 
un nombre très élevé de PME et d'ETI, pas systématiquement des filiales de ces groupes, sont très actives dans ces filières et vont se développer grâce à leur capacité à innover et à exporter.

Le couplage taille/secteur fait ressortir :

- le secteur de l'eau, très concentré autour des grandes entreprises et de leurs filiales avec trois opérateurs détenant $75 \%$ du marché privé de l'eau en France ;

- le secteur de la gestion des déchets, sur fond de faible croissance des marchés de la collecte due à la réduction des volumes et aux nouveaux enjeux de la valorisation, est majoritairement piloté par une douzaine de groupes de 500 à 2000 salariés offrant une bonne couverture du territoire ;

- le secteur de la récupération, très largement le domaine des PME avec $60 \%$ du CA, dont la moitié dans la récupération des fers et métaux ;

- le secteur des services qui compte des dizaines de bureaux d'études techniques et cabinets, voire d'experts indépendants ;

- les secteurs du génie écologique et des énergies renouvelables, les plus récents et les plus dynamiques, avec 100000 emplois et 10 milliards d'euros de CA, représentés par des entreprises structurées et donc porteuses du tissu éco-industriel français du futur. À titre d'exemple, la croissance moyenne de ces sociétés a été de l'ordre de $20 \%$ sur ces dix dernières années et devrait se poursuivre en dépit des politiques tarifaires et d'orientations changeantes.

Ainsi donc, si une partie des métiers de l'environnement est ancienne, tels les métiers de l'eau et des déchets, une nouvelle vague d'entreprises est très récente ; c'est en particulier le cas des entreprises spécialistes des pollutions, des GES (Gaz à Effet de Serre), du solaire et de l'éolien ; on peut signaler, pour ces derniers, la création en 2010 de la plate-forme Windustry, forte déjà de 300 acteurs.

\subsubsection{Attentes vis-à-vis des partenaires-fournisseurs équipementiers-ensembliers}

Les entreprises de la mécanique peuvent être considérées comme des entreprises connexes dont le cour de métier n'est pas l'environnement, mais qui ont développé des compétences dans le secteur.

A côté des sociétés de services et d'ingénieries, les industriels de la mécanique, de la mécatronique et de l'automation associée peuvent considérer l'environnement comme une diversification.

- Secteur de l'eau et de l'assainissement:

- entreprises du forage et du captage dont les besoins en outillages neufs et rénovés sont très importants (outils de coupe, trépans, carburiers, traitements thermiques...);

- entreprises fabriquant des équipements pour la construction et le fonctionnement des stations, du transport de l'eau jusqu'aux technologies 
intégrées dans les installations de traitement (filtre-presse, réducteurs, agitateurs, pompes, décanteurs, chaudronnerie...) ;

- entreprises de maintenance et services associés de travaux.

Une enquête du MEDDE de 2012 précise : $65 \%$ de TPE, $30 \%$ de PME, $5 \%$ relevant des GE.

- Secteur des déchets, valorisation, recyclage et valorisation énergétique :

- entreprises fabricantes d'équipements pour le tri et la sélection des matières (broyeurs, cribleurs, tamis et métal déployé, brûleurs...) ;

- entreprises fabricantes de grands équipements pour l'incinération et la récupération d'énergie, biogaz (incinérateurs, chaudières biomasse, méthaniseurs, torches plasma...).

- Secteur des énergies renouvelables :

- entreprises fabriquant des machines-outils à destination des chaînes de fabrication ;

- entreprises fabriquant des modules, composants ou systèmes pour panneaux solaires, éoliennes, installations géothermiques ;

- entreprises fabriquant de grandes turbines (> $5 \mathrm{MW}$ ) ;

- entreprises «smart grids» ou intervenant dans les réseaux intelligents de consommation électrique (compteurs...) ;

- entreprises de l'aéraulique...

Le COSEI (Comité Stratégique de Filières Éco-Industries) créé en 2008 a vocation à réunir tous les acteurs concernés par le développement des écoindustries ; il est intégré dans un dispositif plus vaste, le Conseil National de l'Industrie, qui vise à relancer l'activité industrielle française.

\subsubsection{Domaines clés de recherche et innovation}

Dans le domaine de l'environnement et des industries liées, les niveaux de maturité sont très différents puisqu'à côté des métiers et technologies mûrs comme ceux de l'eau et de la collecte des déchets, d'autres métiers sont en pleine émergence, ce qui est particulièrement vérifié dans le solaire ou la valorisation des métaux stratégiques.

Il est désormais certain qu'un processus inéluctable de transformation de nos économies, de nos modes de production et de consommation est enclenché.

La transition écologique et énergétique n'en est qu'à ses débuts. Cela nécessite une forte adaptation des mentalités et des appareils productifs, avec un effort d'innovation et une envie de conquête des marchés mondiaux des éco-industries par la mécanique.

Le sujet de l'innovation fait partie justement des groupes de travail transversaux mis en place au COSEI pour améliorer la compétitivité des écoentreprises. Une illustration en est proposée dans un schéma extrait du Livre blanc du PEXE (association des écoentreprises de France, à l'origine Plan EXport des Écoentreprises) : « Financement public de l'innovation par filières et domaines 2011 ». 


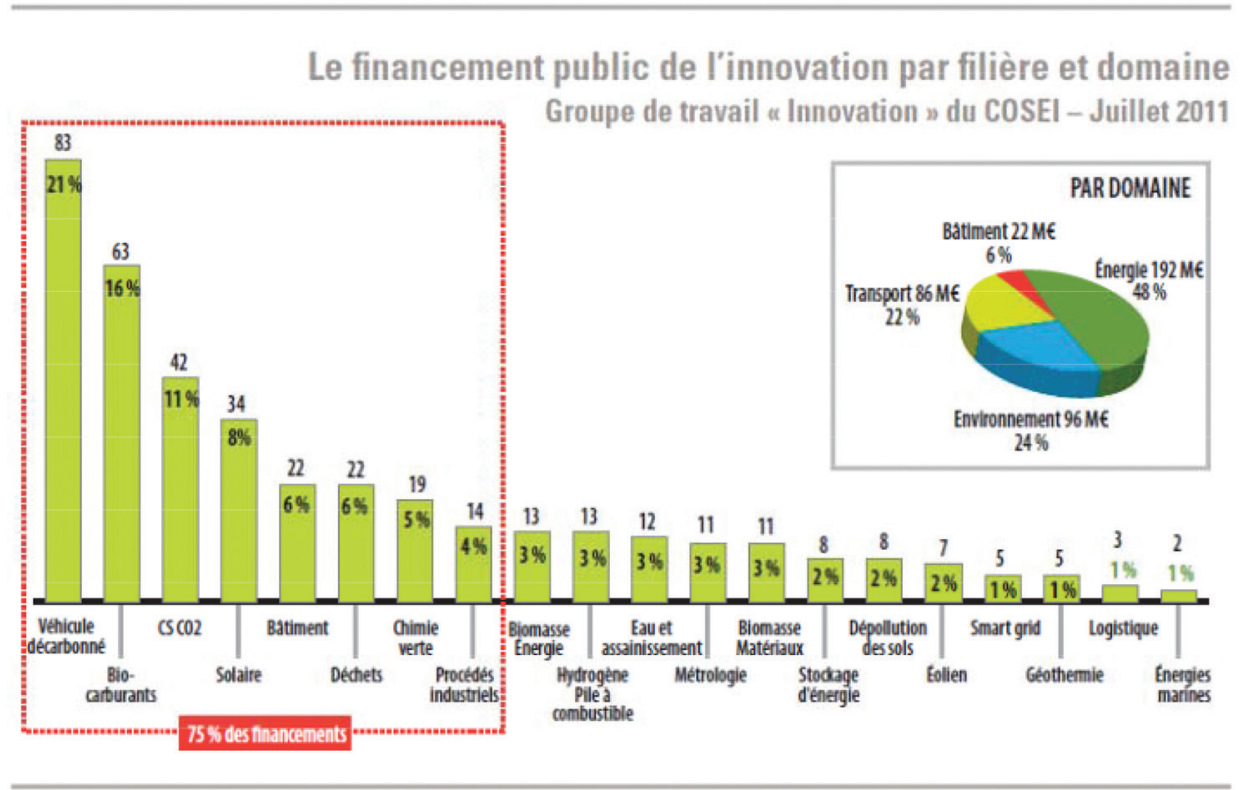

Figure 2.12. - Le financement public des innovations des éco-entreprises.

Parmi les pôles de compétitivité, 14 d'entre eux font de la thématique environnementale leur cœur de cible.

Le réseau Ecotech rassemble ces 14 pôles autour d'une charte commune afin, entre autres, de renforcer les écosystèmes d'innovation favorables à la croissance.

Au niveau de la trajectoire de développement des PME, deux types de développement se dessinent :

- l'hyperspécialisation pour devenir champion dans l'activité particulière ; le levier de croissance est prioritairement l'innovation, suivie de l'exportation ;

- la croissance externe ou l'adjonction de compétences connexes pour répondre à des besoins de plus en plus transverses.

Six domaines d'action stratégiques ont été définis comme prioritaires et nous privilégions en ce qui nous concerne :

- les impacts environnementaux : eau, air, sol, bruit, odeur et adaptation au changement climatique ;

- la métrologie et l'instrumentation des milieux de l'environnement ;

- les matières premières, secondaires et l'économie circulaire.

Parmi les initiatives à mettre en avant :

- la labellisation "cluster d'excellence » et le réseau écoentreprises E2IA en Auvergne ;

- les rencontres Ecotech des instituts Carnot et le PEXE; 
- les projets d'industrialisation en valorisation de métaux stratégiques dont l'Europe n'est pas productrice ;

- les technologies émergentes fruits de collaborations industrie/recherche académique et/ou centres techniques de ressources de la profession, principalement le CETIM : fluides en conditions supercritiques, torches à plasma...

Île-de-France, Rhône-Alpes, PACA et Languedoc-Roussillon sont les régions les plus dynamiques et denses en France. Cela s'explique par le fait qu'elles rassemblent l'expertise historique, les pôles de compétitivité, les entreprises innovantes et les instituts de recherche et universitaires, toujours selon le PEXE.

\subsubsection{Bibliographie}

[1] MEDDE, statistiques.developpement-durable.gouv.fr.

[2] PEXE, Livre blanc.

[3] INSEE, ÉSANE.

[4] ADEME, « Marchés et emplois des activités liées aux déchets ».

[5] CODESPAR, www.codespar.org.

[6] SYVED, Syndicat de la valorisation et de l'élimination de déchets.

[7] ECHA, European Chemicals Agency, echa.europa.eu. 